Raffael Pereira Cezar Zamper

\title{
ASSOCIAÇÃO ENTRE TERAPIA GUIADA POR TESTES VISCOELÁSTICOS COM CONCENTRADOS DE FATORES SINTÉTICOS E TRANSFUSÃO DE SANGUE ALOGÊNICA NO TRANSPLANTE DE FíGADO: um estudo antes-depois
}

Tese apresentada à Faculdade Israelita de Ciências da Saúde Albert Einstein para obtenção do Título de Doutor em Ciências da Saúde. 
Raffael Pereira Cezar Zamper

\section{ASSOCIAÇÃO ENTRE TERAPIA GUIADA POR TESTES VISCOELÁSTICOS COM CONCENTRADOS DE FATORES SINTÉTICOS E TRANSFUSÃO DE SANGUE ALOGÊNICA NO TRANSPLANTE DE FíGADO: um estudo antes-depois}

Tese apresentada à Faculdade Israelita de Ciências da Saúde Albert Einstein para obtenção do Título de Doutor em Ciências da Saúde.

Orientador: Prof. Dr. Ary Serpa Neto 
Z26a

Zamper, Raffael Pereira Cezar

Associação entre terapia guiada por testes viscoelásticos com concentrados de fatores sintéticos e transfusão de sangue alogênica no transplante de fígado : um estudo antes-depois / Raffael Pereira Cezar Zamper. -- São Paulo, 2020.

$\mathrm{xi}, 39 \mathrm{f}$

Tese (Doutorado) - Faculdade Israelita de Ciências da Saúde Albert Einstein. Instituto Israelita de Ensino e Pesquisa Albert Einstein. Programa de Pós-Graduação em Ciências da Saúde.

Título em inglês: Association between viscoelastic tests-guided therapy with synthetic fator concentrates and allogenic blood transfusion in liver transplantation: a before-after study.

1. Transplante de fígado. 2. Transtornos da coagulação sanguínea. 3. Transfusão de sangue. 4. Hemostasia. 5. Tromboelastografia.

NLM - WI 770

Elaborada pelo Sistema Einstein Integrado de Bibliotecas 
FACULDADE ISRAELITA DE CIÊNCIAS DA SAÚDE ALBERT EINSTEIN

Coordenador do Curso de Pós-Graduação: Prof. Dr. Luiz Vicente Rizzo 


\title{
ASSOCIAÇÃO ENTRE TERAPIA GUIADA POR TESTES VISCOELÁSTICOS COM CONCENTRADOS DE FATORES SINTÉTICOS E TRANSFUSÃO DE SANGUE ALOGÊNICA NO TRANSPLANTE DE FíGADO: um estudo antes-depois
}

\author{
BANCA EXAMINADORA
}

Presidente da banca: Prof. Dr. Ary Serpa Neto

Membros titulares:

Prof. Dr. Joao Carlos de Campos Guerra

Prof. Dr. Luiz Fernando dos Reis Falcão

Profa. Dra. Laís Helena Navarro Lima

Membros suplentes:

Prof. Dr. Helio Halpern

Profa. Dra. Maria José Carmona

Aprovada em: 05/08/2020. 


\section{Dedicatória}

Ao Prof. Dr. Flavio Takaoka, meu grande mentor, que durante toda minha carreira sempre esteve ao meu lado, me estimulando, defendendo e aconselhando. Sua vida profissional é um exemplo a ser seguido, e seus valores e princípios serão um grande legado para as futuras gerações de anestesiologistas. 


\section{Agradecimentos}

Primeiramente, ao meu orientador, Prof. Dr. Ary Serpa Neto, por ter aceito me orientar apesar da área diferente. Exímio pesquisador, presente em todos os momentos, ensinando e estimulando de diversas formas, se tornou um grande amigo acima de tudo.

Aos meus pais, Kleny e Romeu, por tudo que me ensinaram, por toda a luta desde a minha infância para que tivesse sucesso na vida pessoal e profissional.

À minha avó, Maria Rosa, e à memória do meu avô, Ordelão, que sempre estiveram ao meu lado, desde criança, complementando a minha formação como ser humano, adicionando princípios e valores à minha vida.

À minha esposa, Simone, e às nossas duas filhas, Julia e Helena, por terem entendido que apesar de o trabalho consumir um tempo grande de minha vida e que a Pós-Graduação adicionaria mais tempo longe delas, era um projeto fundamental para meu futuro profissional, e por isso compreenderam e sempre me apoiaram.

Aos Drs. Thiago Chaves Amorim, Veronica Neves Fialho, Jordana Dantas de Oliveira Lira e Luiz Guilherme Villares da Costa, que me auxiliaram durante a fase de coleta e análise de dados, facilitando o desenvolvimento do projeto.

À Takaoka Anestesia, principalmente aos meus sócios, que suportaram o projeto, entenderam que muitas vezes eu precisaria estar fora da assistência clínica para me dedicar a ele, e que isso significaria trabalho adicional para todos eles.

Por último, a todos os profissionais do Programa de Transplantes do Hospital Israelita Albert Einstein, pela confiança e parceria, na luta constante para que possamos melhorar as condições de cuidados e qualidade de vida dos nossos pacientes. 


\section{Sumário}

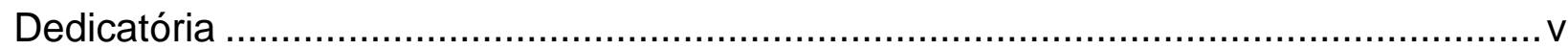

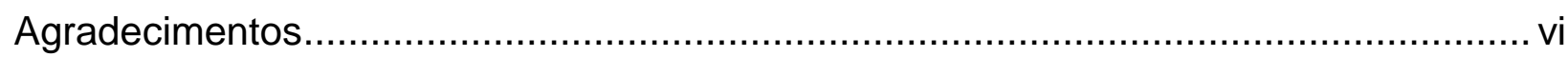

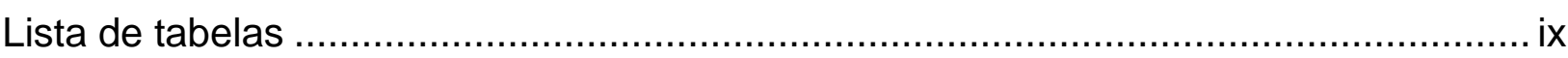

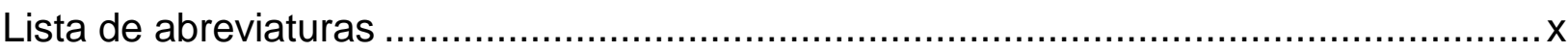

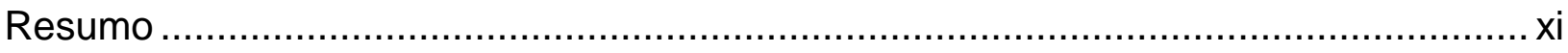

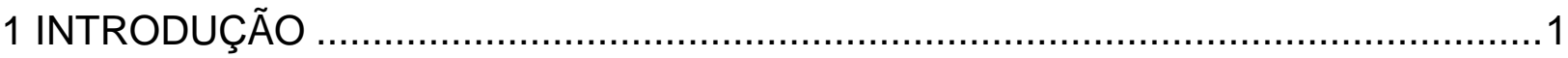

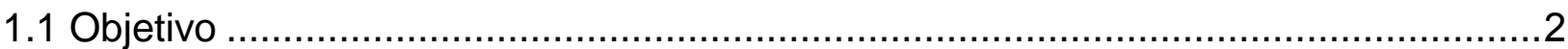

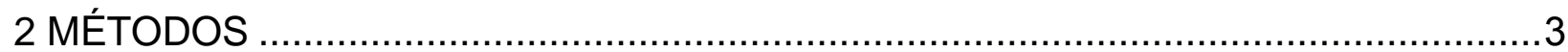

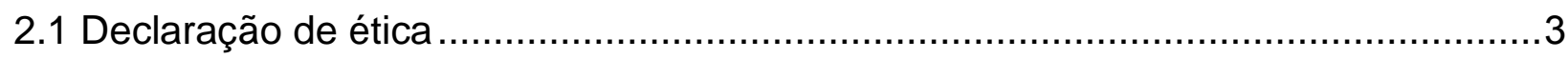

2.2 Pacientes e local de coleta dos dados ..........................................................

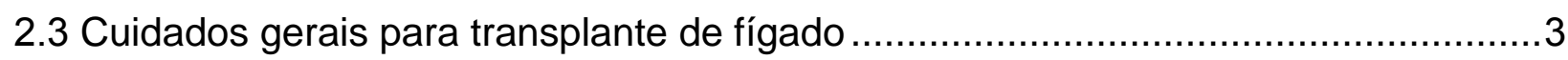

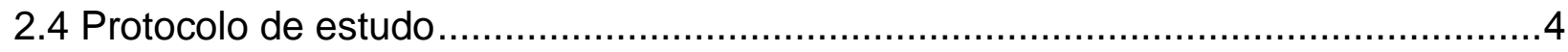

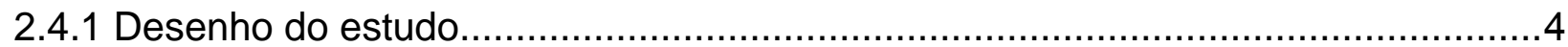

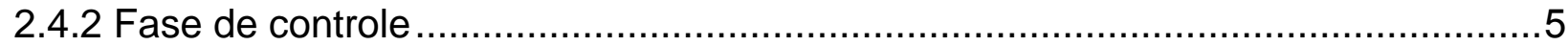

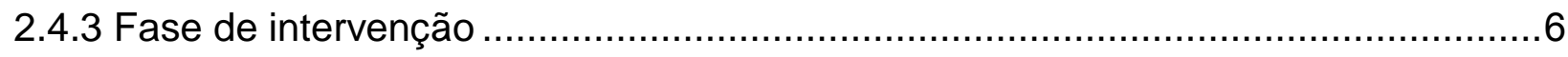

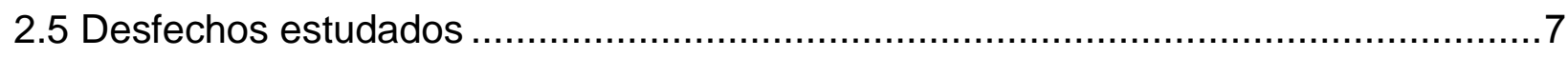

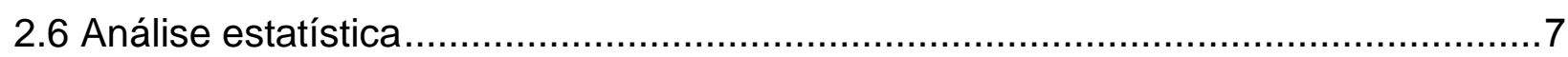

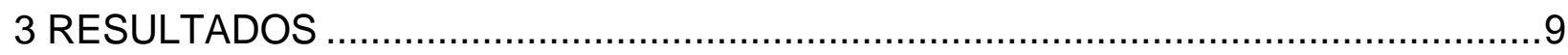

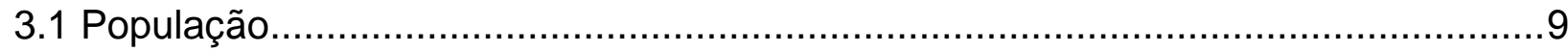

3.2 Características pós-operatórias e cirúrgicas................................................... 12

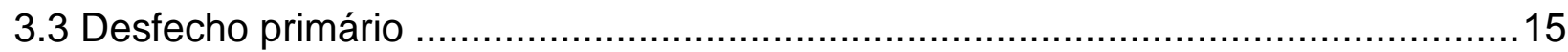

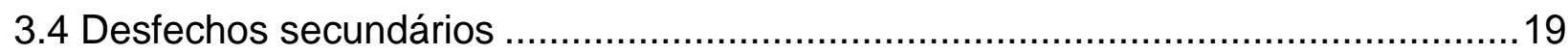

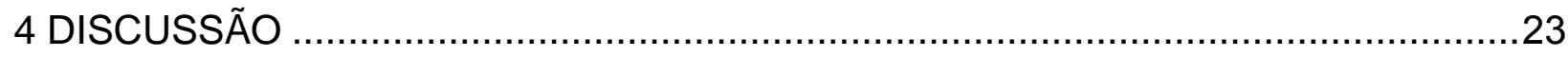

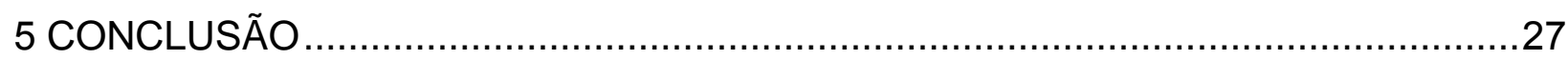

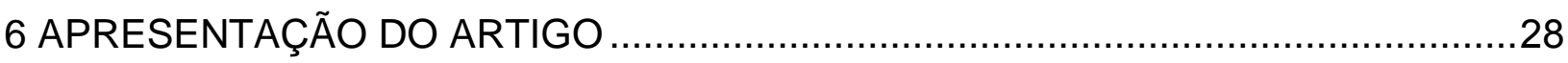

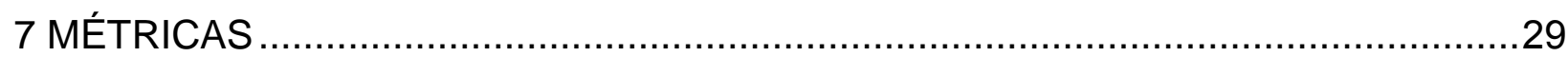

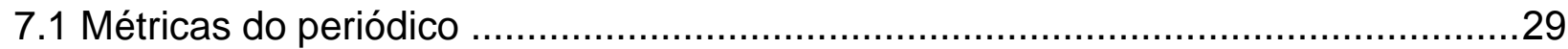

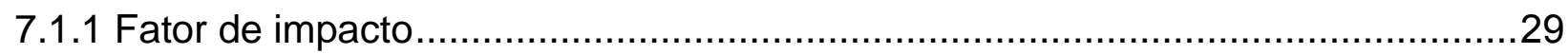

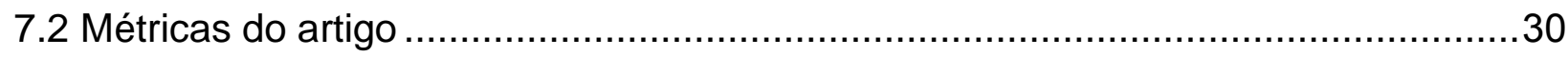

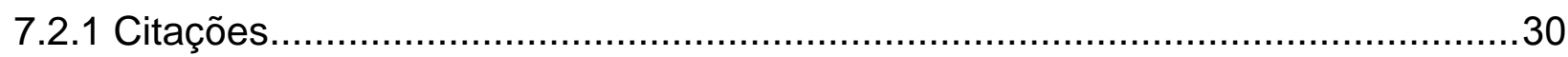

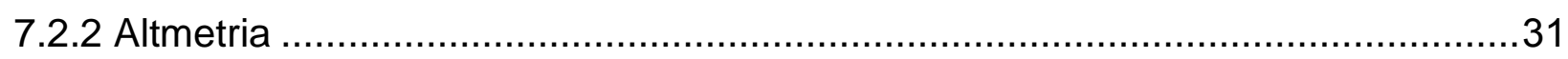

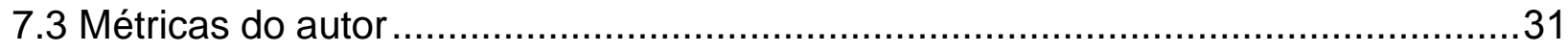


7.3.1 Redes científicas 31

7.3.2 Trabalhos publicados .32

7.3.3 Mediana do fator de impacto dos trabalhos publicados. 32

7.3.4 Citações recebidas .33

7.3.5 H índex e m quociente. .33

8 REFERÊNCIAS .35

Abstract 


\section{Lista de tabelas}

Tabela 1. Características basais dos pacientes .............................................. 10

Tabela 2. Características cirúrgicas e pós-operatórias ......................................... 13

Tabela 3. Transfusão de hemocomponentes ................................................ 16

Tabela 4. Transfusão de hemocomponentes na coorte não pareada .......................17

Tabela 5. Transfusão de hemocomponentes na coorte pareada ...............................18

Tabela 6. Resultados clínicos após o transplante hepático .....................................20

Tabela 7. Resultados clínicos após o transplante hepático na coorte não pareada......21

Tabela 8. Resultados clínicos após o transplante hepático na coorte pareada............22 


\section{Lista de abreviaturas}

A5EX Firmeza do coágulo após 5 minutos em EXTEM

A10 $10_{\mathrm{EX}} \quad$ Firmeza do coágulo após 10 minutos em FIBTEM

CCP Concentrado de complexo protrombínico

CF Concentrado de fibrinogênio

$\mathrm{CH} \quad$ Concentrado de hemácias

$\mathrm{CHC} \quad$ Carcinoma hepatocelular

CLI30 EX Índice de lise do coágulo após 30 minutos em EXTEM

$\mathrm{CT}_{\mathrm{EX}} \quad$ Tempo de coagulação no EXTEM

CT HEP $_{\text {Hempo de coagulação no HEPTEM }}$

$\mathrm{CT}_{\text {IN }} \quad$ Tempo de coagulação no INTEM

MELD Model of End-stage Liver Disease

PFC Plasma fresco congelado

RNI Razão Normalizada Internacional

ROTEM Tromboelastometria de rotação

TACO Sobrecarga circulatória associada à transfusão (Transfusion associated circulatory overload)

TEG Tromboelastografia

TP Tempo de protrombina

TRALI Transfusion-related acute lung injury

TTPA Tempo de tromboplastina parcialmente ativada

UTI Unidade de terapia intensiva 


\section{Resumo}

Introdução: Sangramentos e transfusões perioperatórias são importantes causas de morbimortalidade em pacientes submetidos a transplante de fígado. Objetivo: Avaliar se a terapia guiada por testes viscoelásticos com o uso de hemoderivados afeta as taxas de transfusão de hemocomponentes em pacientes adultos submetidos a transplante de fígado. Métodos: Estudo comparativo intervencionista antes e depois. Os pacientes submetidos a transplante de fígado antes da implementação de um protocolo utilizando tromboelastometria e hemoderivados foram comparados com os pacientes após a implementação. $O$ desfecho primário foi a transfusão de quaisquer hemocomponentes. Os desfechos secundários incluíram: transfusão de concentrado de hemácias, plasma fresco congelado, crioprecipitado ou plaquetas, complicações clínicas, tempo de internação e mortalidade hospitalar. Resultados: Obtivemos 183 pacientes na fase de controle e 54 na fase de intervenção. Após a correspondência do escore de propensão, a proporção de pacientes que receberam qualquer transfusão de hemocomponentes foi menor na fase de intervenção ( 37,0 versus $58,4 \%$; OR 0,42 ; IC95\% 0,20-0,87; $p=0,019)$. Os pacientes na fase de intervenção receberam menos hemácias ( 30,2 versus $52,5 \%$; OR 0,21; IC95\% 0,08-0,56; $\mathrm{p}=0,002)$ e PFC $(5,7$ versus $27,3 \%$; OR 0,11 ; IC95\% $0,03-0,43 ; p=0,002$ ). Não houve diferença em relação à transfusão de crioprecipitado e plaquetas, complicações relacionadas ao procedimento, tempo de internação hospitalar e mortalidade. Conclusões: $\mathrm{O}$ uso de um algoritmo de transfusão viscoelástico guiado por teste com o uso de hemoderivados reduz as taxas de transfusão de sangue alogênico em pacientes submetidos a transplante de fígado.

Descritores: Transplante de fígado; Transtornos da coagulação sanguínea;

Transfusão de sangue; Hemostasia; Tromboelastografia 


\section{INTRODUÇÃo}

O sangramento perioperatório é uma das causas mais importantes de morbimortalidade no transplante de fígado. ${ }^{(1)}$ No entanto, a transfusão sanguínea, usada para corrigir hemorragias e coagulopatias, está diretamente associada a aumento da incidência de infecções, ao aumento de complicações respiratórias, a maior tempo de permanência em unidade de terapia intensiva (UTI), ao maior tempo de internação hospitalar e à maior taxa de reoperações. ${ }^{(2-4)}$ Mesmo assim, mais da metade dos pacientes submetidos a transplante de fígado necessitam de transfusão de algum hemocomponente no período perioperatório. ${ }^{(5-11)}$

Pacientes com doença hepática avançada geralmente apresentam alterações na coagulação e hemostasia. Testes convencionais como tempo de protrombina (TP), tempo de tromboplastina parcialmente ativada (TTPA), contagem de plaquetas e dosagem sérica de fibrinogênio frequentemente mostram valores anormais que apontam para um estado de hipocoagulabilidade. ${ }^{(12)}$ No entanto, testes mais sofisticados demonstram que esses pacientes geralmente apresentam alterações concomitantes nas vias pró e anti-coagulante, resultando em um novo estado de equilíbrio hemostático. ${ }^{(12)}$

O período perioperatório do transplante de fígado é caracterizado por alterações dinâmicas causadas pelo trauma cirúrgico extenso, com produção reduzida de fatores de coagulação e distúrbios fisiopatológicos secundários a trauma anestésico e cirúrgico no paciente cirrótico. ${ }^{(1)}$ Essas alterações podem resultar em sangramento maciço, o que geralmente leva a um tratamento baseado em deficiências mais prováveis ou em resultados laboratoriais tardios que não correspondem mais à situação clínica real. De fato, estudos recentes enfatizam que os testes de coagulação convencionais apresentam limitações significativas nesse cenário, como maior tempo para fornecer resultados úteis, ausência de correlação com o risco de sangramento intraoperatório e falta de avaliação de fatores anticoagulantes, fibrinólise e disfunção plaquetária. ${ }^{(13-17)}$

A tromboelastografia (TEG ${ }^{\circledR}$, Haemoscope / Haemonetics, Niles, III) é utilizada no transplante de fígado desde a década de $1980 .{ }^{(18)} \mathrm{A}$ tromboelastometria rotacional $\left(\mathrm{ROTEM}^{\circledR}\right)$ adota os mesmos princípios do $\mathrm{TEG}^{\circledR}$, como método point-of-care que avalia as propriedades viscoelásticas do sangue total, permitindo a avaliação das 
fases de iniciação, formação, estabilização e lise do coágulo. ${ }^{(18)}$ Estudos recentes demonstraram que a avaliação da coagulação e a terapia guiada por testes viscoelásticos durante procedimentos de alto risco, como cirurgia cardiovascular e do trauma, podem ter um impacto significativo na redução da transfusão de hemocomponentes e também na morbimortalidade dos pacientes. ${ }^{(19,20)}$

Alguns estudos apoiam o uso de testes viscoelásticos no manejo da coagulação perioperatória no transplante de fígado, ${ }^{(21,22)}$ acrescentando informações valiosas em tempo real durante as diferentes etapas da cirurgia. No entanto, as estratégias baseadas nesses testes ainda estão em desenvolvimento e os gatilhos para transfusão de hemocomponentes são desconhecidos. Além disso, a adoção do Model of End-stage Liver Disease (MELD) como método de alocação de órgãos em locais com baixa oferta de doadores faz com que os pacientes sejam submetidos ao transplante de fígado em um estágio muito avançado da doença, o que pode levar a taxas mais altas de transfusão durante o procedimento. De fato, o impacto da terapia guiada por testes viscoelásticos nesse cenário ainda não está claro.

\subsection{Objetivo}

1. Avaliar se a terapia guiada por testes viscoelásticos com o uso de hemoderivados (concentrado de fibrinogênio e concentrado de complexo protrombínico) está associada à diminuição da transfusão de hemocomponentes para correção de distúrbios da coagulação em pacientes adultos submetidos ao transplante hepático em hospital privado no Brasil, utilizando MELD como método de alocação de órgãos. 


\section{MÉTODOS}

\subsection{Declaração de ética}

O protocolo foi aprovado pelo comitê de ética local do Hospital Israelita Albert Einstein (São Paulo, Brasil). Consentimento informado foi dispensado no grupo prospectivo pois trata-se de um processo de melhoria dentro de um programa colaborativo de melhoria da qualidade institucional aplicado a todos os pacientes submetidos a transplante de fígado no hospital. Número CAAE: 33307514.6.0000.0071. Os custos relacionados ao projeto foram pagos pelo centro de custos do Programa de Transplantes do Hospital Israelita Albert Einstein.

\subsection{Pacientes e local de coleta dos dados}

O presente estudo foi realizado na sala de cirurgia e na UTI de um hospital particular. Dados de pacientes adultos submetidos ao transplante de fígado foram coletados e analisados. Todos os pacientes submetidos ao transplante hepático cadavérico por doença hepática crônica foram considerados para inclusão. Não foram incluídos os pacientes com transplante devido à insuficiência hepática aguda, idade $<18$ anos, receptores para transplantes combinados (por exemplo, fígado e rim) e aqueles que necessitam de retransplante em menos de trinta dias após o primeiro transplante. Foram excluídos do estudo pacientes que não apresentavam coleta de dados completa ou nos quais a coleta de dados não foi possível.

\subsection{Cuidados gerais para transplante de fígado}

Os pacientes foram admitidos na sala cirúrgica sem receber medicação pré-anestésica e monitorados com eletrocardiograma, oximetria de pulso e índice bispectral (BIS). Acesso venoso de calibre $16 \mathrm{G}$ e linha arterial radial foram inseridos antes da indução anestésica. Após intubação endotraqueal, foi obtido um acesso venoso central preferencialmente na veia jugular interna direita, e todos os 
pacientes foram monitorados com ecocardiograma transesofágico intraoperatório. Em um grupo específico de pacientes, também foi utilizado um cateter de artéria pulmonar (presença de hipertensão pulmonar, cardiomiopatia ou pontuação no MELD acima de 30). O tipo de anestesia (total intravenosa ou balanceada) foi deixado a critério do anestesiologista. Durante todo o período, os mesmos grupos de anestesiologistas e cirurgiões foram responsáveis por todos os transplantes de fígado. Todos os pacientes usaram meias elásticas e um compressor pneumático intermitente nos membros inferiores. Ao final, todos os pacientes foram encaminhados para a UTI.

\subsection{Protocolo de estudo}

\subsubsection{Desenho do estudo}

Foi utilizado um desenho de estudo antes-depois, pois a literatura mostrou que os testes viscoelásticos são superiores, agregando benefícios aos pacientes submetidos ao transplante hepático, e na instituição o ROTEM já era utilizado há alguns anos, então nao podíamos dar um passo atrás e realizar um estudo prospectivo randomizado com dois braços em que um não utilizaria o ROTEM, pois isso poderia trazer malefício aos pacientes e seria eticamente inapropriado.

O período de controle (antes) foi composto por pacientes submetidos ao transplante de fígado operados pelo menos cinco anos antes da implementação de um protocolo utilizando testes viscoelásticos e hemoderivados. Para entender a escolha do grupo controle, é importante entender algumas características do programa de transplantes do Hospital Israelita Alita Albert Einstein: o programa teve início oficialmente em meados de 2002, então nossos controles viriam dessa janela de tempo entre 2002 e o fim de 2014.

No entanto, entre 2002 e 2007 a alocação de órgãos era feita por ordem de chegada na lista, e em 2007 o critério de alocação passou a utilizar o MELD score, que é um score de gravidade, e dessa forma os pacientes mais graves tinham prioridade em serem transplantados. Essa mudança fundamental na alocação de orgãos causou uma mudança sensível no perfil de gravidade dos pacientes transplantados, e decidimos então excluir os pacientes transplantados antes da era MELD como candidatos a controles, evitando um viés de seleção no nosso 
entendimento, pois a gravidade dos pacientes está relacionada com maior ou menor necessidade de transfusão, e era importante que os dois grupos fossem semelhantes do ponto de vista de gravidade.

Reduzida a janela a ser utilizada na base histórica, nos restavam então os transplantes realizados entre 2007 e 2014, mas aqui novamente encontramos uma particularidade: no período entre 2009 e 2014, o ROTEM estava disponível para uso, e era usado de forma heterogênea entre os anestesistas do grupo, sem algoritmo definido; não havia política clara e definida de seu uso nos transplantes hepáticos. Além disso, nesse mesmo período os hemoderivados tornaram-se disponíveis para uso nos pacientes do programa, também sem indicação clara, sem um algoritmo, e não eram usados por todos os anestesiologistas da equipe. Portanto não incluímos os transplantes ocorridos nesse intervalo de tempo, e assim nosso grupo controle reduziuse aos pacientes transplantados entre 2007 e 2009, já na era MELD, e sem o uso do ROTEM e de hemoderivados, evitando assim, potenciais viéses que viriam a poluir nosso resultado.

A fase de intervenção (depois) consistiu em pacientes submetidos a transplante de fígado operados durante um período de 10 meses após essa implementação (janeiro a outubro de 2015).

E esse foi o desenho final do estudo: um estudo antes-depois com o grupo controle retrospectivo composto de pacientes transplantados entre 2007 e 2009, e o grupo intervenção prospectivo composto por pacientes transplantados num período de 10 meses entre janeiro e outubro de 2015.

\subsubsection{Fase de controle}

$\mathrm{Na}$ fase de controle, a estratégia de transfusão e a escolha do produto sanguíneo foram realizadas a critério do anestesiologista responsável pelo caso. O ROTEM ${ }^{\circledast}$ não estava disponível durante esse período. Foi realizada uma análise retrospectiva dos dados gravados prospectivamente sobre características demográficas, exames laboratoriais, medicamentos, características cirúrgicas, estratégia de reposição hídrica, uso de hemocomponentes, sinais vitais, complicações após a cirurgia, tempo de ventilação mecânica no período pós-operatório, tempo de internação na UTI, tempo total de hospitalização e mortalidade intra-hospitalar. 


\subsubsection{Fase de intervenção}

Durante um período de 10 meses, o procedimento recomendado foi uma estratégia de tratamento da coagulopatia intraoperatória baseada nos resultados dos testes viscoelásticos com uso predominantemente de hemoderivados. Os pacientes foram acompanhados até a alta hospitalar ou óbito, o que ocorresse primeiro.

A transfusão foi indicada quando havia coagulopatia e sangramento evidentes, com base em um algoritmo projetado em conjunto com hematologistas e especialistas na área (Figura 1). O ROTEM ${ }^{\circledR}$, colhido da linha arterial em tubos dedicados e enviados imediatamente ao laboratório do Hospital Israelita Albert Einstein, foi realizado nos seguintes momentos: no período pré-operatório dos exames laboratoriais de rotina na enfermaria, 15 minutos após reperfusão arterial, seis horas após o término do transplante na UTI e a qualquer momento em que a equipe considerasse necessário com base em aspectos clínicos do campo cirúrgico.

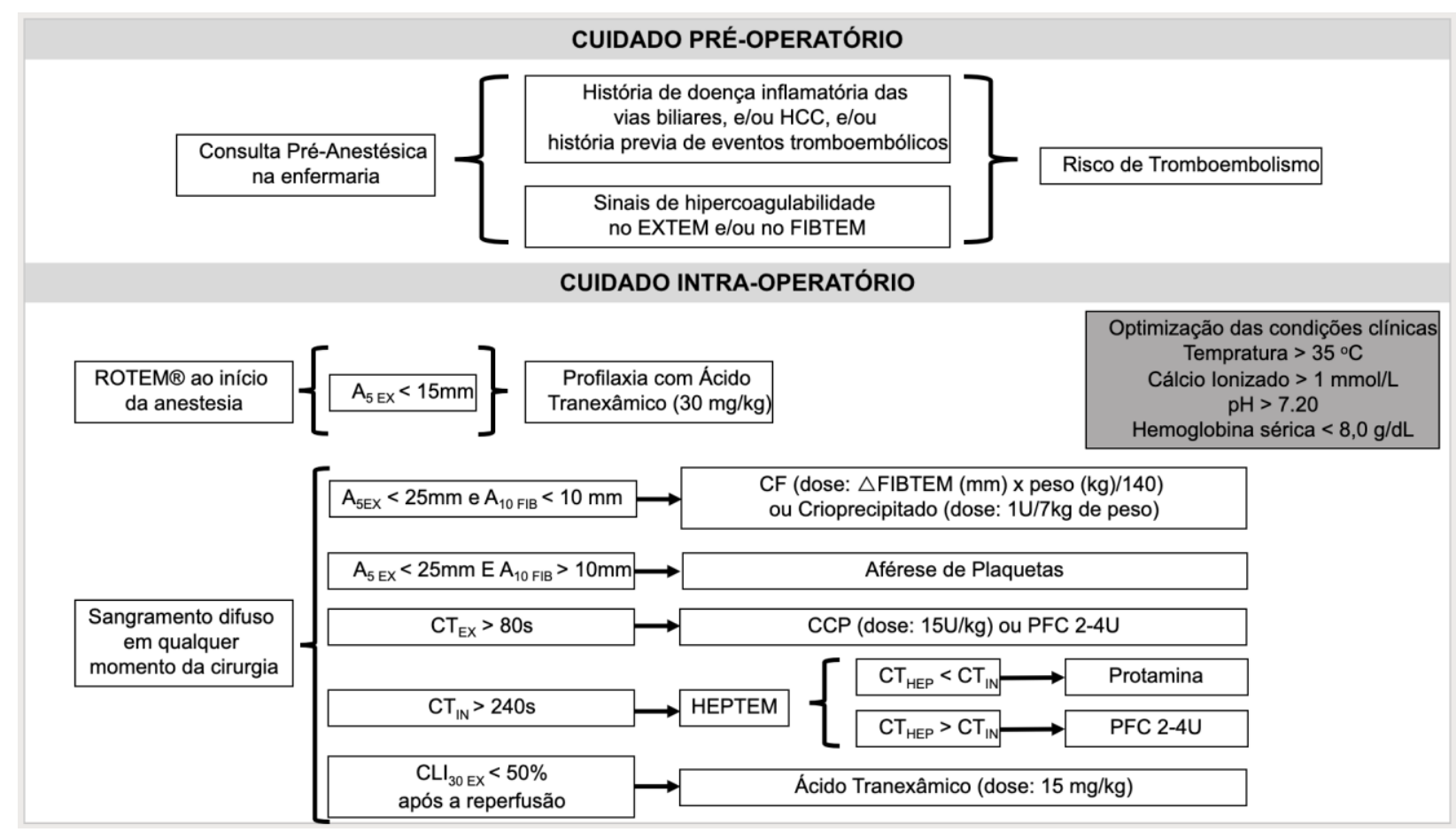

Figura 1. Algoritmo para avaliação e tratamento da alteração da coagulação durante o transplante de fígado 


\subsection{Desfechos estudados}

O desfecho primário foi o número total de unidades de transfusão de quaisquer hemocomponentes, definidos como concentrado de hemácias $(\mathrm{CH})$, plasma fresco congelado (PFC), crioprecipitado ou plaquetas. Os desfechos secundários estudados foram:

1) Transfusão de $\mathrm{CH}$;

2) Transfusão de PFC;

3) Transfusão de crioprecipitado;

4) Transfusão de plaquetas;

5) Uso de hemoderivados ou antifibrinolítico;

6) Complicações relacionadas ao procedimento (definidas como hemorragia digestiva alta e/ou trombose arterial);

7) Duração pós-operatória de ventilação mecânica em dias;

8) Tempo de internação em UTI e tempo de internação hospitalar em dias;

9) Mortalidade intra-hospitalar.

\subsection{Análise estatística}

A fase de controle foi definida para uma duração de 5 anos e a fase de intervenção para 10 meses a priori. A relação número de controles para número de intervenções foi definida como 3:1. De acordo com os resultados, o presente estudo tem um poder de $77 \%$ para detectar diferenças no resultado primário com um erro tipo I de $5 \%$.

A análise primária consistiu em comparar qualquer transfusão de hemocomponentes entre as duas fases por meio de um teste do qui-quadrado. Para considerar o viés potencial associado ao desenho antes-depois, realizamos duas análises com um ajuste para características demográficas. Inicialmente, uma análise multivariada foi realizada usando um modelo de regressão logística ou linear, incluindo variáveis que diferem entre as duas fases nas análises bivariadas e aquelas que já são conhecidas como fatores prognósticos no transplante de fígado. As variáveis utilizadas para o ajuste foram idade, escore MELD, classificação de Child-Pugh, presença de 
carcinoma hepatocelular $(\mathrm{CHC})$ e níveis pré-operatórios de albumina, ureia, creatinina e hemoglobina. Após isso, um método de escore de propensão foi aplicado para equilibrar covariáveis nas duas fases e reduzir o viés. Os escores de propensão foram estimados para cada paciente com regressão logística usando idade, classificação de Child-Pugh, escore de MELD, presença de CHC e níveis pré-operatórios de hemoglobina como covariáveis. O escore de propensão reflete a propensão no intervalo de 0 a 1 para estar na fase de intervenção, devido a um conjunto de variáveis conhecidas, e é uma tentativa de ajustar o possível viés de seleção, fatores de confusão e diferenças entre os grupos. Pacientes com dados ausentes foram excluídos do banco de dados. Com base nos estimadores ponderados do escore de propensão para os dados, construímos uma coorte correspondente ao escore de propensão. $O$ pareamento foi realizado com o vizinho mais próximo, sem substituição, com cada paciente da fase de intervenção pareado com dois pacientes da fase de controle. Uma largura do compasso de calibre de 0,1 do desvio padrão do logit do escore de propensão foi usada para o desenvolvimento da correspondência. Uma regressão logística ou linear foi realizada nesta amostra combinada. Todos os resultados são apresentados como odds ratio (OR) e intervalo de confiança de $95 \%$ (IC95\%) para regressão logística ou o coeficiente $\beta$ e IC95\% para regressão linear.

A normalidade das variáveis foi testada com o teste de KolmogorovSmirnoff. Os dados paramétricos contínuos foram expressos como a média e os dados não paramétricos foram expressos em mediana e intervalo interquartil. Os dados categóricos foram expressos em números absolutos e porcentagem. Para características demográficas, foi utilizado o teste $t$ de Student, conforme apropriado. Todas as análises foram realizadas com o SPSS v.20 (IBM SPSS Statistics for Windows, versão 20.0. Armonk, NY: IBM Corp.) e R v.2.12.0. Para todas as análises, foram considerados significativos valores-p bilateral $\mathrm{e}<0,05$. 


\section{RESULTADOS}

\subsection{População}

As características demográficas da população incluída são relatadas na tabela 1. Cento e oitenta e três pacientes foram incluídos no controle e cinquenta e quatro na fase de intervenção. No início do estudo, os pacientes na fase de intervenção apresentaram menor incidência de doença renal crônica, encefalopatia e hemorragia digestiva alta, menores níveis pré-operatórios de creatinina e ureia e maiores níveis de albumina. 
Tabela 1. Características basais dos pacientes

\begin{tabular}{|c|c|c|c|c|c|c|}
\hline Características basais e comorbidades & \multicolumn{3}{|c|}{$\begin{array}{l}\text { Coorte não pareada } \\
(\mathrm{n}=237)\end{array}$} & \multicolumn{3}{|c|}{$\begin{array}{l}\text { Coorte pareada } \\
(n=135)^{b}\end{array}$} \\
\hline Sexo, masculino & $43 / 54(79,6)$ & $131 / 183(71,6)$ & 0,239 & $35 / 46(76,1)$ & $71 / 89(79,8)$ & 0,62 \\
\hline Peso, kg & $77,8 \pm 15,9$ & $76,0 \pm 17,0$ & 0,483 & $76,2 \pm 16,5$ & $76,7 \pm 15,7$ & 0,867 \\
\hline Altura, cm & $171,2 \pm 8,0$ & $168,3 \pm 9,9$ & 0,051 & $170,2 \pm 7,8$ & $170,1 \pm 8,6$ & 0,927 \\
\hline $\mathrm{IMC}, \mathrm{kg} / \mathrm{m}^{2}$ & $26,6 \pm 4,4$ & $26,7 \pm 5,1$ & 0,848 & $26,3 \pm 4,6$ & $26,4 \pm 4,6$ & 0,945 \\
\hline Doença renal crônica & $3 / 54(5,6)$ & $34 / 183(18,6)$ & 0,02 & $2 / 46(4,3)$ & $17 / 89(19,1)$ & 0,019 \\
\hline Hipertensão & $14 / 54(25,9)$ & $31 / 183(16,9)$ & 0,139 & $13 / 46(28,3)$ & $14 / 89(15,7)$ & 0,084 \\
\hline Diabetes mellitus & $11 / 54(20,4)$ & $48 / 183(26,2)$ & 0,381 & $9 / 46(19,6)$ & $28 / 89(31,5)$ & 0,141 \\
\hline Etiologia de doença hepática & & & 0,578 & & & 0,852 \\
\hline Budd-Chiari & $0 / 54(0,0)$ & $3 / 182(1,6)$ & & $0 / 46(0,0)$ & $1 / 89(1,1)$ & \\
\hline Alcoólica & 14 / $54(25,9)$ & $32 / 182(17,6)$ & & $11 / 46(23,9)$ & $22 / 89(24,7)$ & \\
\hline Hepatite C & $23 / 54(42,6)$ & $89 / 182(48,9)$ & & $19 / 46(41,3)$ & $40 / 89(44,9)$ & \\
\hline PAF & $0 / 54(0,0)$ & $5 / 182(2,7)$ & & $0 / 46(0,0)$ & $0 / 89(0,0)$ & \\
\hline Falência hepática aguda & $0 / 54(0,0)$ & $2 / 182(1,1)$ & & $0 / 46(0,0)$ & $1 / 89(1,1)$ & \\
\hline Outros & $6 / 54(11,1)$ & $10 / 182(5,5)$ & & $6 / 46(13,0)$ & $6 / 89(6,7)$ & \\
\hline \multicolumn{7}{|l|}{ Status clínico pré-transplante } \\
\hline Uso de ventilação mecânica & $2 / 54(3,7)$ & $14 / 183(7,7)$ & 0,309 & $2 / 46(4,3)$ & $6 / 89(6,7)$ & 0,576 \\
\hline Uso de hemodiálise & $4 / 54(7,4)$ & $16 / 183(8,7)$ & 0,756 & $3 / 46(6,5)$ & $7 / 89(7,9)$ & 0,777 \\
\hline Cirurgia prévia & $8 / 54(14,8)$ & 37 / $183(20,2)$ & 0,373 & $8 / 46(17,4)$ & $14 / 89(15,7)$ & 0,804 \\
\hline Classificação de Child-Pugh & & & 0,019 & & & 0,223 \\
\hline A & $13 / 54(24,1)$ & $44 / 183(24,0)$ & & $13 / 46(28,3)$ & $17 / 89(19,1)$ & \\
\hline $\mathrm{B}$ & $7 / 54(13,0)$ & $57 / 183(31,1)$ & & $7 / 46(15,2)$ & $24 / 89(27,0)$ & \\
\hline C & 34 / $54(63,0)$ & $82 / 183(44,8)$ & & $26 / 46(56,5)$ & 48 / $89(53,9)$ & \\
\hline
\end{tabular}




\begin{tabular}{|c|c|c|c|c|c|c|}
\hline Escore de MELD & $22.7 \pm 8,8$ & $21.6 \pm 8,2$ & 0,418 & $21.9 \pm 9,2$ & $22.0 \pm 7,9$ & 0,954 \\
\hline Re-transplante & $1 / 54(1,9)$ & $0 / 183(0,0)$ & 0,065 & $1 / 46(2,2)$ & $0 / 89(0,0)$ & 0,162 \\
\hline \multicolumn{7}{|l|}{ Complicações } \\
\hline Encefalopatia & $1 / 54(1,9)$ & $94 / 183(51,4)$ & $<0,001$ & $1 / 46(2,2)$ & $50 / 89(56,2)$ & $<0,001$ \\
\hline Trombose da veia porta & 4 / $54(7,4)$ & $10 / 183(5,5)$ & 0,594 & $3 / 46(6,5)$ & 4 / $89(4,5)$ & 0,614 \\
\hline Hipertensão pulmonar & $1 / 54(1,9)$ & $4 / 183(2,2)$ & 0,88 & $1 / 46(2,2)$ & 2 / $89(2,2)$ & 0,978 \\
\hline $\mathrm{CHC}$ & $23 / 54(42,6)$ & $53 / 183(29,0)$ & 0,059 & $16 / 46(34,8)$ & $28 / 89(31,5)$ & 0,696 \\
\hline Refratária & 14 / $54(25,9)$ & $78 / 183(42,6)$ & & $11 / 46(23,9)$ & $35 / 89(39,3)$ & \\
\hline \multicolumn{7}{|l|}{ Medicações em uso } \\
\hline Furosemida & 22 / $54(40,7)$ & $53 / 183(29,0)$ & 0,101 & $20 / 46(43,5)$ & $27 / 89(30,3)$ & 0,128 \\
\hline Espironolactona & $27 / 54(50,0)$ & $68 / 183(37,2)$ & 0,013 & $23 / 46(50,0)$ & 37 / $89(41,6)$ & 0,35 \\
\hline Propranolol & $27 / 54(50,0)$ & $58 / 183(31,7)$ & 0,09 & 22 / $46(47,8)$ & $33 / 89(37,1)$ & 0,228 \\
\hline \multicolumn{7}{|c|}{ Testes Laboratoriais pré-transplante } \\
\hline $\mathrm{RNI}$ & $1,8 \pm 0,8$ & $1,7 \pm 0,6$ & 0,113 & $1,8 \pm 0,8$ & $1,7 \pm 0,7$ & 0,532 \\
\hline Fibrinogênio, mg/dL & $214,5 \pm 92,6$ & $178,3 \pm 94,5$ & 0,301 & $214,5 \pm 92,6$ & $153,7 \pm 70,2$ & 0,095 \\
\hline Plaquetas, x1000/mm ${ }^{3}$ & $80,5 \pm 59,2$ & $74,2 \pm 55,0$ & 0,473 & $81,6 \pm 62,7$ & $67,1 \pm 48,7$ & 0,143 \\
\hline Ureia, mg/dL & $36,5 \pm 15,7$ & $49,7 \pm 43,3$ & 0,03 & $35,9 \pm 14,9$ & $51,9 \pm 47,8$ & 0,028 \\
\hline Creatinina, $\mathrm{mg} / \mathrm{dL}$ & $0,9 \pm 0,4$ & $1,2 \pm 0,8$ & 0,004 & $0,9 \pm 0,4$ & $1,2 \pm 0,7$ & 0,008 \\
\hline Sódio, mEq/L & $137,1 \pm 3,3$ & $137,5 \pm 5,8$ & 0,702 & $137,3 \pm 3,0$ & $137,4 \pm 6,1$ & 0,963 \\
\hline Potássio, mEq/L & $4,3 \pm 0,5$ & $4,1 \pm 0,5$ & 0,074 & $4,3 \pm 0,5$ & $4,2 \pm 0,5$ & 0,316 \\
\hline Fração de ejeção do VE, \% & $66,2 \pm 8,5$ & $67,0 \pm 7,3$ & 0,577 & $66,4 \pm 8,9$ & $68,3 \pm 6,6$ & 0,253 \\
\hline
\end{tabular}

Kg: kilogramas; cm: centímetros; IMC: índice de massa corpórea; CEP: colangite esclerosante primária; MELD: Model for End-Stage Liver Disease; PAF: Polineuropatia Amilóide Familial; RNI: razão normalizada internacional; mg: miligramas; dL; decilitros; g: gramas; CHC: carcinoma hepatocelular; VE: ventrículo esquerdo. Dados apresentados como média \pm desvio padrão ou número/total (porcentagem). ${ }^{a}$ Comparação de diferenças entre os dois grupos usando o teste t para variáveis contínuas e o teste $x^{2}$ para variáveia categóricas. ${ }^{b}$ Ajustado por idade, Child, MELD, presença de CHC e hemoglobina sérica pré-transplante. 


\subsection{Características pós-operatórias e cirúrgicas}

Os tempos de clampeamento e de isquemia foram menores na fase de intervenção (Tabela 2). No entanto, em ambas as fases, esses tempos permaneceram dentro do ideal. No intraoperatório, os pacientes na fase de intervenção receberam menos flúidos e apresentaram menor balanço hídrico. Ao final da cirurgia, os pacientes em fase de intervenção apresentaram menor frequência cardíaca, pressão venosa central e temperatura e maior pressão arterial média e dose de noradrenalina (Tabela 2). 
Tabela 2. Características cirúrgicas e pós-operatórias

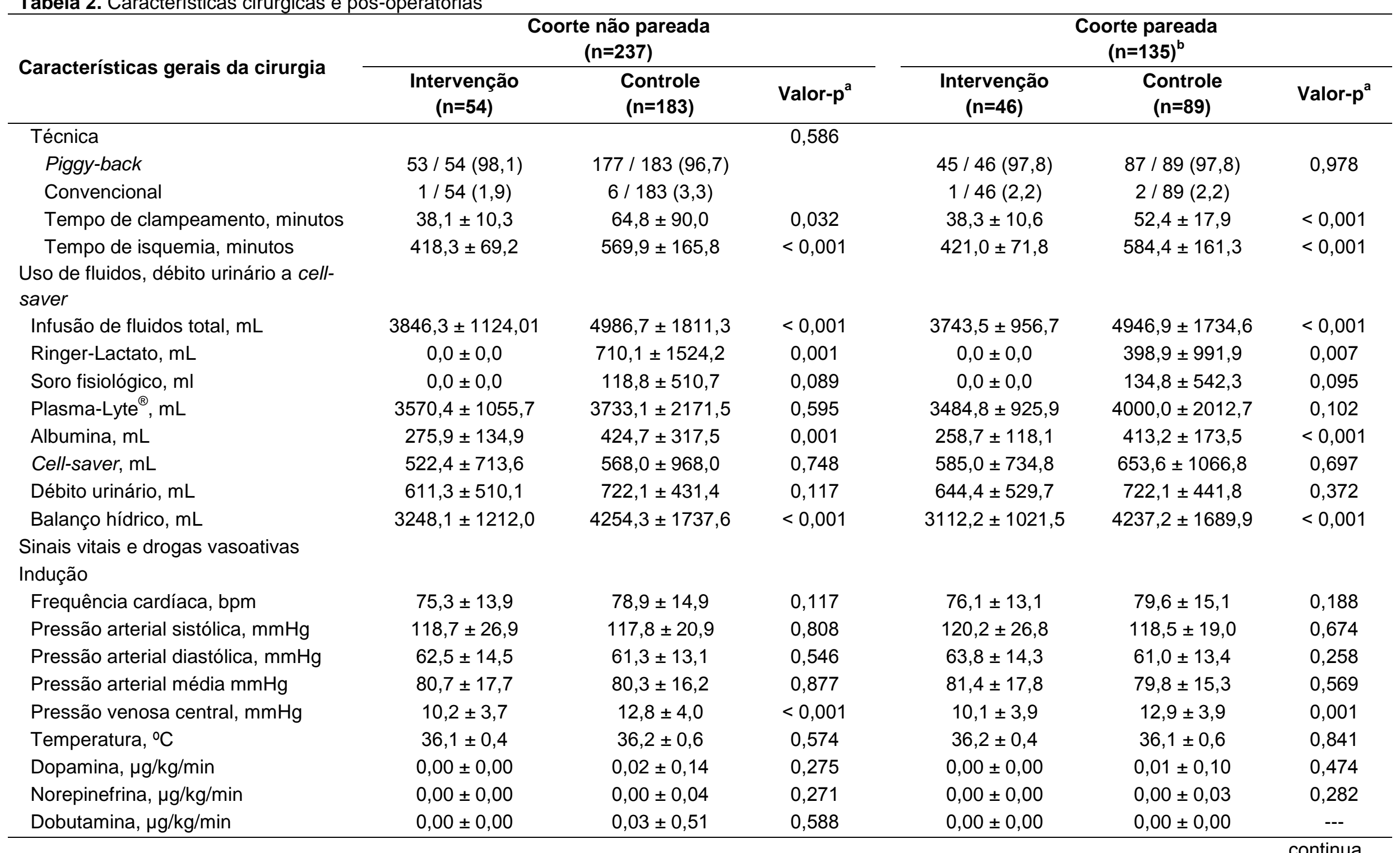




\begin{tabular}{|c|c|c|c|c|c|c|}
\hline \\
\hline \multicolumn{7}{|l|}{$\begin{array}{l}\text { Ao final } \\
\text { Frequência cardíaca, bpm }\end{array}$} \\
\hline Pressão arterial sistólica, mmHg & $118,4 \pm 20,7$ & $113,4 \pm 16,3$ & 0,063 & $117,7 \pm 21,6$ & $114,2 \pm 16,7$ & 0,297 \\
\hline Pressão arterial diastólica, $\mathrm{mmHg}$ & $61,5 \pm 11,4$ & $57,0 \pm 11,0$ & 0,01 & $60,6 \pm 10,7$ & $57,2 \pm 11,2$ & 0,092 \\
\hline Pressão arterial média mmHg & $80,4 \pm 13,8$ & $76,1 \pm 12,9$ & 0,036 & $80,0 \pm 13,9$ & $76,5 \pm 12,1$ & 0,127 \\
\hline Pressão venosa central, mmHg & $9,3 \pm 4,7$ & $11,0 \pm 2,7$ & 0,001 & $9,6 \pm 4,4$ & $10,9 \pm 2,9$ & 0,043 \\
\hline Temperatura, ${ }^{\circ} \mathrm{C}$ & $36,1 \pm 0,6$ & $37,5 \pm 0,5$ & $<0,001$ & $36,1 \pm 0,6$ & $37,5 \pm 0,6$ & $<0,001$ \\
\hline Dopamina, $\mu \mathrm{g} / \mathrm{kg} / \mathrm{min}$ & $0,00 \pm 0,00$ & $0,01 \pm 0,13$ & 0,346 & $0,00 \pm 0,00$ & $0,00 \pm 0,00$ & --- \\
\hline Norepinefrina, $\mu \mathrm{g} / \mathrm{kg} / \mathrm{min}$ & $0,06 \pm 0,12$ & $0,02 \pm 0,05$ & $<0,001$ & $0,06 \pm 0,11$ & $0,02 \pm 0,04$ & $<0,001$ \\
\hline Dobutamina, $\mu \mathrm{g} / \mathrm{kg} / \mathrm{min}$ & $0,13 \pm 1,02$ & $0,29 \pm 1,24$ & 0,392 & $0,16 \pm 1,10$ & $0,24 \pm 1,16$ & 0,686 \\
\hline
\end{tabular}

$\mathrm{mL}$ : millilitros; bpm: batimentos por minuto. Dados apresentados como média \pm desvio padrão ou número/total (porcentagem). ${ }^{2}$ Comparação de diferenças entre os dois grupos usando teste t para variáveis contínuas e teste $x^{2}$ para variáveis categóricas. ${ }^{b}$ Ajustado por idade, Child, MELD, presença de CHC e hemoglobina sérica pré-transplante. 


\subsection{Desfecho primário}

A proporção de pacientes que receberam qualquer transfusão de hemocomponentes foi de $35,2 \%$ na fase de intervenção e $56,3 \%$ na fase de controle $(p=0,006)$ (Tabela 3). Ao considerar o ajuste para potenciais fatores de confusão, os pacientes na fase de intervenção ainda apresentavam menor risco de transfusão de hemocomponentes em comparação aos da fase de controle (OR ajustado, 0,25; IC $95 \%, 0,10-0,63 ; p=0,003$ ) (Tabela 4). Após a correspondência do escore de propensão, a proporção de pacientes que receberam qualquer transfusão de hemocomponentes ainda era menor na fase de intervenção (37,0 versus $58,4 \%$; $p=0,018 ;$ OR $=0,42 ;$ IC95\% 0,20 - 0,87; $p=0,019$ ) (Tabelas 3 e 5). 
Tabela 3. Transfusão de hemocomponentes

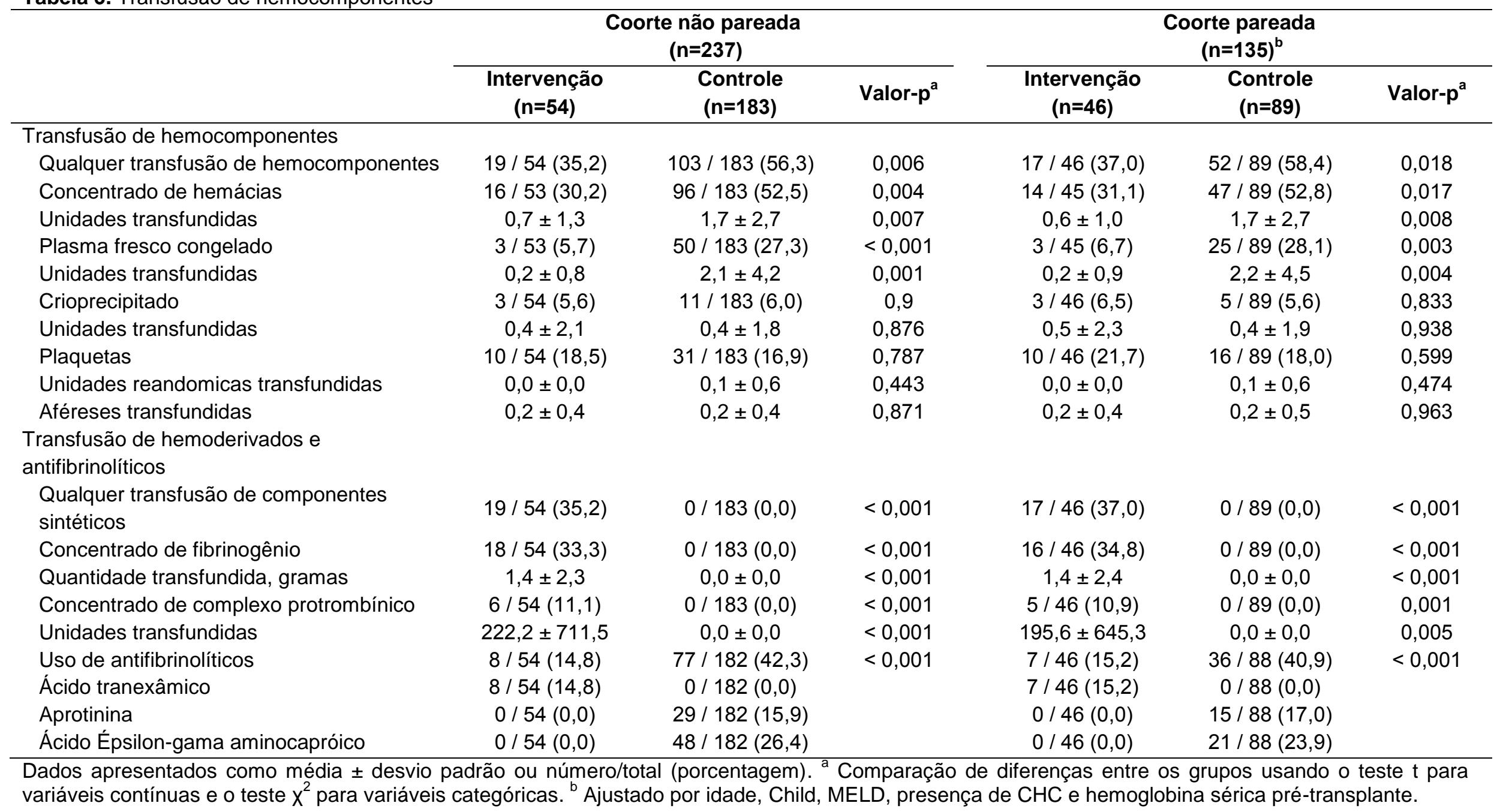


Tabela 4. Transfusão de hemocomponentes na coorte não pareada

\begin{tabular}{|c|c|c|c|c|c|}
\hline & \multicolumn{3}{|c|}{$\begin{array}{c}\text { Coorte não pareada } \\
(n=237)\end{array}$} & \multicolumn{2}{|c|}{ Regressão logística ajustada ${ }^{b}$} \\
\hline \multicolumn{6}{|c|}{ Transfusão de hemocomponentes } \\
\hline $\begin{array}{l}\text { Qualquer transfusão de } \\
\text { hemocomponentes }\end{array}$ & $19 / 54(35,2)$ & $103 / 183(56,3)$ & 0,006 & $0,25(0,10-0,63)$ & 0,003 \\
\hline Concentrado de hemácias & 16 / $53(30,2)$ & $96 / 183(52,5)$ & 0,004 & $0,21(0,08-0,56)$ & 0,002 \\
\hline Plasma fresco congelado & $3 / 53(5,7)$ & $50 / 183(27,3)$ & $<0,001$ & $0,11(0,03-0,43)$ & 0,002 \\
\hline \multicolumn{6}{|l|}{$\begin{array}{l}\text { Transfusão de componentes } \\
\text { sintéticos }\end{array}$} \\
\hline $\begin{array}{l}\text { Qualquer transfusão de } \\
\text { componentes sintéticos }\end{array}$ & $19 / 54(35,2)$ & $0 / 183(0,0)$ & $<0,001$ & --- & --- \\
\hline Concentrado de fibrinogênio & $18 / 54(33,3)$ & $0 / 183(0,0)$ & $<0,001$ & --- & --- \\
\hline $\begin{array}{l}\text { Concentrado de complexo } \\
\text { protrombínico }\end{array}$ & $6 / 54(11,1)$ & $0 / 183(0,0)$ & $<0,001$ & --- & --- \\
\hline
\end{tabular}


Tabela 5. Transfusão de hemocomponentes na coorte pareada

\begin{tabular}{|c|c|c|c|c|c|}
\hline & \multicolumn{3}{|c|}{$\begin{array}{l}\text { Coorte não pareada } \\
(n=237)\end{array}$} & \multicolumn{2}{|c|}{ Regressão logística ajustada ${ }^{\mathrm{b}}$} \\
\hline $\begin{array}{l}\text { Qualquer transfusão de } \\
\text { hemocomponentes }\end{array}$ & 17 / $46(37,0)$ & $52 / 89(58,4)$ & 0,018 & $0,42(0,20-0,87)$ & 0,019 \\
\hline Concentrado de hemácias & $14 / 45(31,1)$ & $47 / 89(52,8)$ & 0,017 & $0,40(0,19-0,86)$ & 0,019 \\
\hline Plasma fresco congelado & $3 / 45(6,7)$ & $25 / 89(28,1)$ & 0,003 & $0,18(0,05-0,64)$ & 0,008 \\
\hline \multicolumn{6}{|l|}{ Transfusão de componentes sintéticos } \\
\hline $\begin{array}{l}\text { Qualquer transfusão de componentes } \\
\text { sintéticos }\end{array}$ & 17 / $46(37,0)$ & $0 / 89(0,0)$ & $<0,001$ & --- & --- \\
\hline Concentrado de fibrinogênio & $16 / 46(34,8)$ & $0 / 89(0,0)$ & $<0,001$ & --- & --- \\
\hline $\begin{array}{l}\text { Concentrado de complexo } \\
\text { protrombínico }\end{array}$ & $5 / 46(10,9)$ & $0 / 89(0,0)$ & 0,001 & --- & --- \\
\hline
\end{tabular}

Dados apresentados como número/total (porcentagem). ${ }^{a}$ Comparação de diferenças entre os dois grupos usando o teste $x^{2} .{ }^{b}$ Ajustado por idade, Child, MELD, presença de CHC, hemoglobina sérica pré-transplante, albumina, ureia e creatinina. Fase controle com referência. 


\subsection{Desfechos secundários}

Os resultados secundários são fornecidos nas tabelas 3 e 6 . Os pacientes na fase de intervenção receberam menos concentrados de hemácias $(30,2$ versus $52,5 \% ; p=0,004$; OR ajustado 0,$21 ; \mathrm{IC} 95 \% 0,08-0,56 ; p=0,002)$ e PFC $(5,7$ versus 27,3\%; $p<0,001$; OR ajustado, 0,11; IC95\%, 0,03 - 0,43; $p=0,002$ ) (Tabelas 3 e 4). Não houve diferença em relação à transfusão de crioprecipitado e plaquetas. O uso de hemoderivados foi maior na fase de intervenção $(35,2$ versus $0,0 \% ; p<0,001)$ e 0 uso de antifibrinolítico menor (14,8 versus 42,3\%; $p<0,001$; OR ajustado 0,33; IC95\% 0,13-0,80; $p=0,015$ ) (Tabelas 3 e 4). Os resultados após a correspondência do escore de propensão produziram os mesmos resultados (Tabelas 3 e 5).

Não houve diferença em relação às complicações relacionadas ao procedimento, tempo de ventilação mecânica, tempo de internação na UTI e mortalidade hospitalar entre os dois grupos (Tabelas 6 e 7). No entanto, o tempo de permanência hospitalar nos sobreviventes foi menor na fase de intervenção $(11,3 \pm 7,2$ versus $16,3 \pm 12,7$ dias; $p=0,007$; coeficiente $\beta$ ajustado, $-5,84 ;$ IC95\%, -9,77 - -1,91; $p=0,004$ ) (Tabelas 6 e 7). Após a correspondência do escore de propensão, houve apenas uma tendência de diminuição do tempo de permanência hospitalar nos sobreviventes na fase de intervenção $(11,6 \pm 7,5$ versus $15,1 \pm 11,4$ dias; $p=0,066$; coeficiente $\beta$ ajustado, -3,53; IC95\%, -7,22 - 0,17; $p=0,061$ ) (Tabelas 6 e 8). 
Tabela 6. Resultados clínicos após o transplante hepático

\begin{tabular}{|c|c|c|c|c|c|c|}
\hline Resultados clínicos & \multicolumn{3}{|c|}{$\begin{array}{l}\text { Coorte não pareada } \\
(n=237)\end{array}$} & \multicolumn{3}{|c|}{$\begin{array}{l}\text { Coorte pareada } \\
(n=135)^{b}\end{array}$} \\
\hline Qualquer complicação & $25 / 53(47,2)$ & $99 / 183(54,1)$ & 0,373 & $21 / 45(46,7)$ & 44 / $89(49,4)$ & 0,761 \\
\hline Hemorragia digestiva alta & $10 / 53(18,9)$ & $54 / 174(31,0)$ & 0,084 & $10 / 45(22,2)$ & $27 / 84(32,1)$ & 0,235 \\
\hline Trombose arterial & $1 / 53(1,9)$ & $6 / 172(3,5)$ & 0,557 & $1 / 45(2,2)$ & $2 / 82(2,4)$ & 0,938 \\
\hline \multicolumn{7}{|l|}{ Geral } \\
\hline Sobreviventes & $0,4 \pm 1,1$ & $0,8 \pm 1,2$ & 0,052 & $0,4 \pm 1,1$ & $0,8 \pm 1,4$ & 0,094 \\
\hline Tempo de permanência na UTI, dias & $3,2 \pm 4,0$ & $4,2 \pm 6,6$ & 0,29 & $3,4 \pm 4,3$ & $3,6 \pm 4,6$ & 0,781 \\
\hline Sobreviventes & $2,8 \pm 2,7$ & $3,6 \pm 5,3$ & 0,306 & $2,9 \pm 2,9$ & $3,5 \pm 4,6$ & 0,463 \\
\hline Tempo de internação hospitalar, dias & $12.1 \pm 8,9$ & $17,2 \pm 15,4$ & 0,022 & $12,4 \pm 9,5$ & $16,1 \pm 16,6$ & 0,172 \\
\hline Sobreviventes & $11,3 \pm 7,2$ & $16,3 \pm 12,7$ & 0,007 & $11,6 \pm 7,5$ & $15,1 \pm 11,4$ & 0,066 \\
\hline Mortalidade intra-hospitalar & $1 / 53(1,9)$ & $11 / 182(6,0)$ & 0,226 & $1 / 45(2,2)$ & $5 / 89(5,6)$ & 0,369 \\
\hline
\end{tabular}

UTI: Unidade de terapia intensiva. Dados apresentados como número/total (porcentagem). ${ }^{2}$ Comparação de diferenças entre os dois grupos usando o teste $X^{2} \cdot{ }^{b}$ Ajustado por idade, Child, MELD, presença de CHC e hemoglobina sérica pré-transplante. 
Tabela 7. Resultados clínicos após o transplante hepático na coorte não pareada

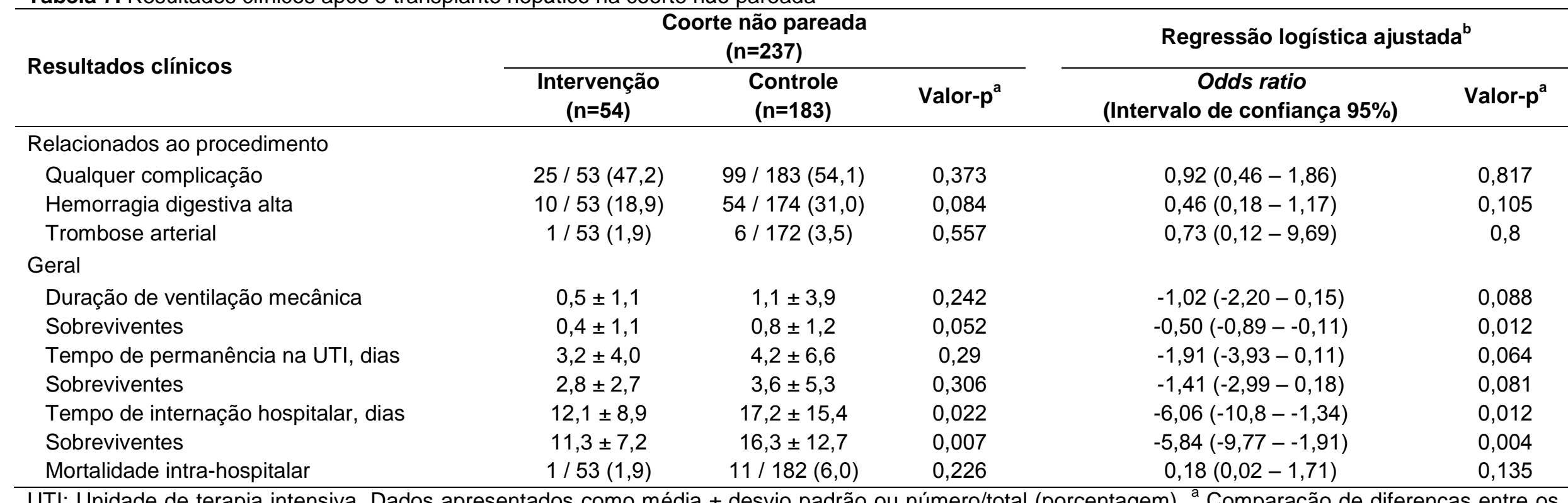

UTI: Unidade de terapia intensiva. Dados apresentados como média \pm desvio padrão ou número/total (porcentagem). ${ }^{a}$ Comparação de diferenças entre os dois grupos usando o teste t para variáveis contínuas e o teste $x^{2}$ para variáveis categóricas. ${ }^{b}$ Ajustado por idade, Child, MELD, presença de CHC, hemoglobina sérica pré-transplante, albumina, ureia e creatinina. Grupo controle como referência. ${ }^{\circ}$ Neste modelo o coeficiente $\beta$ é reportado. 
Tabela 8. Resultados clínicos após o transplante hepático na coorte pareada

\begin{tabular}{|c|c|c|c|c|c|}
\hline Resultados clínicos & \multicolumn{3}{|c|}{$\begin{array}{l}\text { Coorte não pareada } \\
(n=237)\end{array}$} & \multicolumn{2}{|c|}{ Regressão logística ajustada ${ }^{b}$} \\
\hline Qualquer complicação & $21 / 45(46,7)$ & 44 / 89 (49.4) & 0,761 & $0,89(0,44-1,83)$ & 0,762 \\
\hline Hemorragia digestiva alta & $10 / 45(22,2)$ & 27 / $84(32.1)$ & 0,235 & $0,60(0,26-1,39)$ & 0,237 \\
\hline Trombose arterial & $1 / 45(2,2)$ & 2 / $82(2.4)$ & 0,938 & $0,91(0,08-10,31)$ & 0,939 \\
\hline \multicolumn{6}{|l|}{ Geral } \\
\hline Sobreviventes & $0,4 \pm 1,1$ & $0,8 \pm 1.4$ & 0,094 & $-0,41(-0,89-0,06)$ & 0,088 \\
\hline Tempo de permanência na UTI, dias & $3,4 \pm 4,3$ & $3,6 \pm 4.6$ & 0,781 & $-0,23(-1,85-1,38)$ & 0,779 \\
\hline Sobreviventes & $2,9 \pm 2,9$ & $3.5 \pm 4.6$ & 0,463 & $-0,57(-2,08-0,94)$ & 0,458 \\
\hline Tempo de internação hospitalar, dias & $12,4 \pm 9,5$ & $16.1 \pm 16.6$ & 0,172 & $-3,67(-8,86-1,53)$ & 0,166 \\
\hline Sobreviventes & $11,6 \pm 7,5$ & $15.1 \pm 11.4$ & 0,066 & $-3,53(-7,22-0,17)$ & 0,061 \\
\hline Mortalidade intra-hospitalar & $1 / 45(2,2)$ & $5 / 89(5.6)$ & 0,369 & $0,38(0,04-3,37)$ & 0,386 \\
\hline
\end{tabular}

UTI: Unidade de terapia intensiva. Dados apresentados como média \pm desvio padrão ou número/total (porcentagem). ${ }^{a}$ Comparação de diferenças entre os dois grupos usando o teste t para variáveis contínuas e o teste $\chi 2$ para variáveis categóricas. ${ }^{b}$ Após o pareamento por escore de propensão. Fase controle como referência. Neste modelo o coeficiente $\beta$ é reportado. 


\section{DISCUSSÃO}

Neste estudo observacional, a introdução de um algoritmo transfusional baseado no ROTEM ${ }^{\circledR}$ e no uso preferencial de hemoderivados mostrou uma redução acentuada nas taxas de transfusão de quaisquer hemocomponentes, de $\mathrm{CH}$ e PFC isolados, além de uma redução do uso de medicamentos antifibrinolíticos. Nenhum paciente no grupo de tratamento desenvolveu complicações significativas relacionadas ao uso do protocolo.

Nas últimas duas décadas, alguns estudos demonstraram que pacientes cirróticos com aumento da Relação Normalizada Internacional (RNI) podem ter geração normal de trombina ${ }^{(23)}$ e que suas plaquetas são qualitativamente capazes de apresentar boa função frente à geração adequada de trombina quando sua contagem total é de $50-60 \times 10^{9} /$ L. ${ }^{(24)}$ A hemostasia encontra um equilíbrio novo e frágil e os testes laboratoriais convencionais isolados são ineficientes para avaliar a coagulação. ${ }^{(25,26)}$

A decisão de transfundir um paciente submetido a transplante de fígado apresenta-se como um desafio, pois o uso de hemocomponentes está associado a complicações como trombocitopenia e coagulopatia, toxicidade do citrato, distúrbios eletrolíticos e metabólicos, hipotermia, sepse, sobrecarga circulatória associada à transfusão (TACO - transfusion associated circulatory overload) e lesão pulmonar relacionada à transfusão (TRALI - transfusion-related acute lung injury), ${ }^{(27,28)}$ aumentando a mortalidade desses pacientes. ${ }^{(29)}$ Além disso, a transfusão de hemácias aumenta a incidência de trombose da artéria hepática ${ }^{(30)}$ e o uso de crioprecipitado, plaquetas e PFC diminui a sobrevida do enxerto em 1 e 5 anos. ${ }^{(5)}$

O TEG ${ }^{\circledR}$ como método para avaliar a função hemostática global por meio de uma amostra simples de sangue foi descrito em 1948. ${ }^{(31)}$ Desde então, métodos que avaliam as propriedades viscoelásticas do sangue total, como o TEG ${ }^{\circledR}$ e, mais recentemente, o ROTEM ${ }^{\circledR}$, tornaram-se ferramentas complementares aos testes estáticos tradicionais, ${ }^{(17,32)}$ sendo utilizados em cirurgias complexas, como o transplante de fígado, desde o século passado. ${ }^{(18)}$ No entanto, ainda não foram estabelecidos algoritmos baseados em testes viscoelásticos com gatilhos de transfusão bem definidos. 
Este estudo apóia os resultados de estudos anteriores que mostraram a eficácia dos testes viscoelásticos na avaliação e tratamento dos estados de hipocoagulação com sangramento em pacientes sépticos ${ }^{(33)}$ e em cirurgias de alta complexidade, como cirurgia cardiovascular, ${ }^{(34)}$ do trauma ${ }^{(35)}$ e transplante de fígado. ${ }^{(21,22,36,37)} \mathrm{O}$ algoritmo de transfusão proposto, baseado em testes viscoelásticos com gatilhos definidos e associando o uso de hemoderivados como uma opção terapêutica, levou a uma menor taxa de transfusão de componentes sanguíneos. Estudos futuros responderão se essa estratégia melhora a sobrevida do enxerto e do paciente.

O uso de hemoderivados, como o concentrado de fibrinogênio (CF) e o concentrado de complexo protrombínico (CCP) ainda não foi amplamente estudado até o momento no contexto de coagulopatias que requerem tratamento, e um estudo recente mostrou a segurança dessa opção terapêutica em pacientes transplantados hepáticos. ${ }^{(38)} \mathrm{Em}$ nosso estudo, utilizamos hemoderivados predominantemente, uma boa alternativa para substituir o PFC e as transfusões de crioprecipitados, evitando assim as complicações intrínsecas relacionadas à transfusão destes hemocomponentes.

O CF demonstrou-se eficaz no tratamento de pacientes com hipofibrinogenemia em cirurgias obstétricas, ${ }^{(39)}$ cardíacas, ${ }^{(40)}$ e do trauma, ${ }^{(41)}$ melhorando a função do coágulo e reduzindo o sangramento. Alguns autores argumentam que, nas situações em que o crioprecipitado é indicado, a substituição pelo CF oferece vantagens do ponto de vista da eficácia e segurança. ${ }^{(42)}$

Além disso, as alternativas ao tratamento da hipofibrinogenemia são limitadas. O PFC contém quantidades insuficientes de fibrinogênio ${ }^{(43)}$ e é ineficiente nas situações clínicas em que é usado para a reposição de deste. ${ }^{(44)} \mathrm{O}$ crioprecipitado é a terapia de escolha, mas é uma preparação que oferece altos riscos de complicações como transmissão de doenças infecciosas, lesão pulmonar aguda e complicações imuno-mediadas, aumentando a morbimortalidade em pacientes transfundidos. ${ }^{(45-47)}$

É importante notar que, embora não tenhamos encontrado diferença entre as taxas de transfusão de crioprecipitados nos dois grupos, o uso de testes viscoelásticos levou a um aumento na indicação geral de reposição de fibrinogênio no grupo intervenção, o que foi feito com crioprecipitado ou $\mathrm{CF}$, a critério do anestesiologista do caso. Uma explicação possível é que a avaliação mais rápida da 
coagulação ao usar testes viscoelásticos direcionou o tratamento da coagulopatia com reposição adequada. Sabe-se que não há correlação entre valores de exames convencionais como TP ou TTPA e coagulopatia, e que a maioria dos pacientes submetidos a transplante hepático apresenta hipofibrinogenemia intraoperatória. ${ }^{(48,49)}$

O PFC continua sendo a principal terapia para a coagulopatia multifatorial observada no transplante hepático(50-52) e o CCP foi inicialmente apresentado como uma opção para a reversão dos agentes anticoagulantes cumarínicos. $^{(53)}$ Embora não contenha todos os fatores presentes no PFC, uma vez que é compost pelos fatores dependentes da vitamina $\mathrm{K}(\mathrm{II}, \mathrm{VII}, \mathrm{IX}$ e X) e fatores anticoagulantes das proteínas C e S, o CCP contém importantes efetores na coagulação e, portanto, é uma alternativa nos casos em que o PFC é indicado. ${ }^{(54)}$ Apresenta baixo risco de eventos tromboembólicos e oferece a vantagem de menor risco de transmissão de infecções e reações transfusionais quando comparado ao PFC, além de baixo impacto no volume sanguíneo do paciente, reduzindo o risco de sobrecarga de volume e anemias dilucionais. ${ }^{(53,55)} \mathrm{Um}$ estudo recente mostrou que o CCP pode ser mais eficaz que o PFC para restaurar a geração de trombina em pacientes submetidos a transplante de fígado e que a dose necessária é menor que a dose usada para a reversão da varfarina. ${ }^{(56)}$

Os gatilhos transfusionais associados aos testes viscoelásticos não são bem estabelecidos na literatura científica. A maioria dos estudos que propõe um algoritmo baseado em testes viscoelásticos utiliza a avaliação da amplitude no EXTEM no décimo minuto $\left(\mathrm{A} 10_{\mathrm{EX}}\right) .^{(14,36,52,57)} \mathrm{O}$ uso do $\mathrm{A} 5_{\mathrm{EX}}$ já foi mostrado como um parâmetro eficaz para detectar trombocitopenia e hipofibrinogenemia em pacientes submetidos a transplante de fígado. ${ }^{(58)}$ Nosso estudo é inédito ao usar prospectivamente uma avaliação anterior de amplitude no EXTEM no quinto minuto ( $A 5_{E X}$ ), associando-a à amplitude do FIBTEM no décimo minuto $\left(\mathrm{A} 10_{\mathrm{FIB}}\right)$ para apoiar a terapia transfusional, e se esta deve ser realizada com reposição de fibrinogênio ou de plaquetas. Assim, no quinto minuto após o início da formação do coágulo, sabemos se há uma deficiência que precise de tratamento, seja pela transfusão de hemocomponentes ou de hemoderivados.

A hiperfibrinólise é um evento comum em pacientes submetidos a transplante de fígado. É caracterizada pelo desequilíbrio entre ativadores e inibidores do sistema fibrinolítico, responsável por conter a propagação do coágulo, e o resultado desse desequilíbrio é um excesso de degradação da fibrina polimerizada, que gera 
desestabilização do coágulo e sangramento difuso da microvasculatura, sendo uma importante causa de sangramento em pacientes submetidos a transplante hepático. ${ }^{(59)}$ Assim, os antifibrinolíticos são utilizados para reduzir a perda de sangue e a transfusão de hemocomponentes, reduzindo custos e complicações, e a decisão de utilizar esse recurso deve ser individualizada devido ao risco de eventos tromboembólicos. ${ }^{(60,61)}$

A alta incidência de fibrinólise encontrada em pacientes submetidos a transplante de fígado tornou desejável o uso de antifibrinolíticos no passado, excluindo seu uso apenas em pacientes mais propensos a eventos trombóticos, como pacientes com doenças inflamatórias do trato biliar, pacientes com antecedentes de história de eventos trombóticos e pacientes com hepatocarcinoma celular ou outros tipos de câncer. No entanto, foi demonstrado que na maioria das situações em que a fibrinólise está presente em um transplante de fígado, ela é transitória e portanto revertida sem a necessidade de intervenção. ${ }^{(62)}$

A introdução do ROTEM $^{\circledR}$ como ferramenta para avaliação da coagulação permite a identificação de pacientes propensos à fibrinólise e de pacientes com sangramento por fibrinólise, e esses são os pacientes que se beneficiam do uso de antifibrinolíticos. ${ }^{(63,64)}$ Isso explica a redução no uso de antifibrinolíticos observada em nosso estudo, no entanto, não há como avaliar o impacto dessa redução na morbimortalidade.

Nosso estudo tem algumas limitações, a saber o tamanho pequeno da amostra, o desenho realizado em um único centro e o uso de controles não concorrentes. Além disso, os períodos de intervenção e controle ocorreram em diferentes épocas e embora a equipe cirúrgica permaneceu com a mesma composição e utilizando a mesma técnica cirúrgica, entendemos que a curva de aprendizado e maior experiência podem ter contribuído para menores taxas transfusionais no grupo intervenção. Utilizamos o ajuste do escore de propensão na tentativa de controlar alguns desses fatores. Acreditamos que os dados de nosso estudo são internamente consistentes, têm base mecanicista válida e são suportados por outros estudos. 


\section{CONCLUSÃO}

1. A introdução de um algoritmo de tratamento da coagulopatia intraoperatória guiado por testes viscoelásticos e com o uso de hemoderivados em sua linha terapêutica reduz as taxas de transfusão de sangue alogênico em pacientes submetidos ao transplante de fígado. Além disso, observamos redução do uso de concentrados de hemácias e de PFC. Menor necessidade do uso de medicações antifibrinolíticas foi notado. Mais estudos são necessários para identificar se há impacto na morbimortalidade desses pacientes. 


\section{APRESENTAÇÃO DO ARTIGO}

Zamper RP, Amorim TC, Queiroz VN, Lira JD, Costa LG, Takaoka F, Juffermans NP, Serpa Neto A. Association between viscoelastic tests-guided therapy with synthetic factor concentrates and allogenic blood transfusion in liver transplantation: a beforeafter study. BMC Anesthesiol. 2018;18(1):198. doi: 10.1186/s12871-018-0664-8. 


\section{MÉTRICAS}

\subsection{Métricas do periódico}

Métricas do periódico nos últimos cinco anos.

\subsubsection{Fator de impacto}

Periódico: BMC Anesthesiology

ISSN: $1471-2253$

Fator de impacto 2018: 1.619

2018 Journal Impact Factor \& percentile rank in category for: BMC Anesthesiology

\subsection{9}

2018 Journal Impact Factor

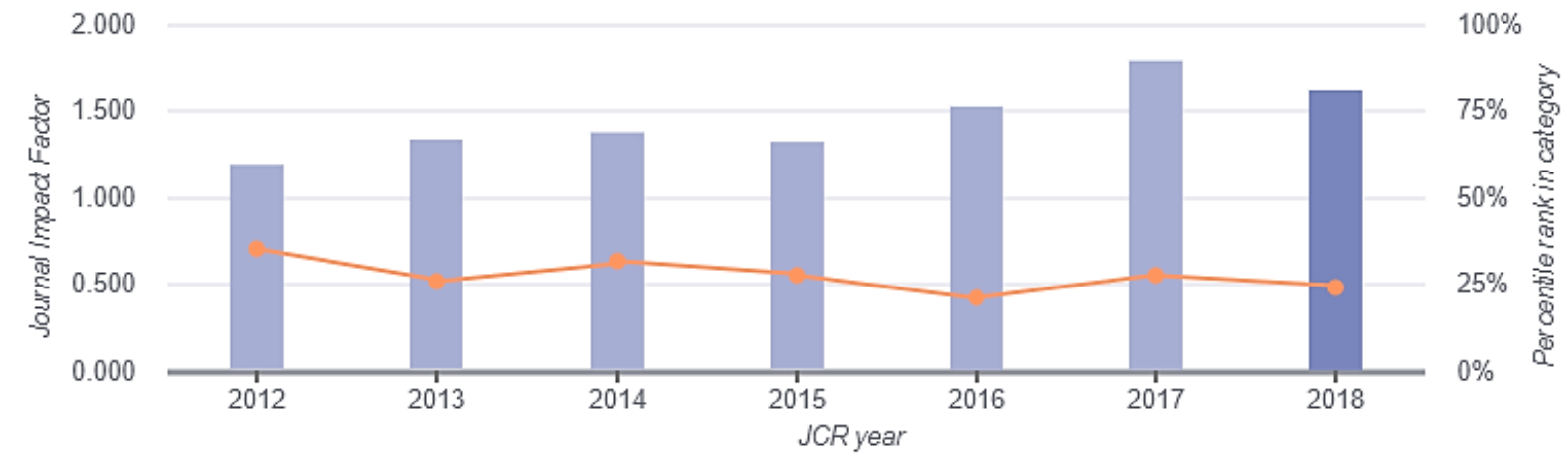

$\square$ JIF $-\odot$ - ANESTHESIOLOGY

Fonte: Journal Citation Reports 


\subsection{Métricas do artigo}

\subsubsection{Citações}

Há uma citação para o artigo nas bases de dados Scopus, Web of Science e Google Scholar, e duas citações em capítulos de livros.

\begin{tabular}{|c|c|c|c|}
\hline Citação recebida & $\begin{array}{c}\text { Scopus/Web of } \\
\text { Science/Google } \\
\text { Scholar }\end{array}$ & $\begin{array}{l}\text { Capítulo de } \\
\text { livro }\end{array}$ & Total \\
\hline $\begin{array}{l}\text { Gorlinger K, Perez-Ferrer A, Dirkmann D, Saner F, } \\
\text { Maegele M, Calatayud AA, et al. The role of } \\
\text { evidence-based algorithms for rotational } \\
\text { thromboelastometry-guided bleeding management. } \\
\text { Korean J Anesthesiol. 2019;72(4):297-322. }\end{array}$ & 1 & - & 1 \\
\hline $\begin{array}{l}\text { Bleszynski MS, Kim PT. Liver transplantation. In: } \\
\text { Rahimi RS, editor. The critically III cirrhotic patient: } \\
\text { Evaluation and management. Cham: Springer } \\
\text { Nature; 2020. p. 273-327. }\end{array}$ & - & 1 & 1 \\
\hline $\begin{array}{l}\text { Karapanagiotou A, Pitsoulis A, Vasileiou M, } \\
\text { Voloudakis N. Management of patients with liver } \\
\text { transplantation in ICU. In: Tsoulfas G, editor; } \\
\text { Rodrigo L, coeditor. Liver disease and surgery. [S.I.]: } \\
\text { IntechOpen; } 2019 \text {. }\end{array}$ & - & 1 & 1 \\
\hline
\end{tabular}

Avaliação realizada em 12 de março de 2020. 


\subsubsection{Altmetria}

Dados altmétricos coletados em 12 de março de 2020.

\begin{tabular}{|c|c|c|}
\hline \multirow[t]{12}{*}{ Escore } & Variáveis & Quantidade \\
\hline & Menções em rede & \\
\hline & Twitter & 3 \\
\hline & Origem das menções & \\
\hline & Alemanha & 2 \\
\hline & Áustria & 1 \\
\hline & Citações & \\
\hline & Dimensions & 3 \\
\hline & Capítulo de livro & 2 \\
\hline & Artigo & 1 \\
\hline & Leituras & 27 \\
\hline & Mendeley & 27 \\
\hline
\end{tabular}

\subsection{Métricas do autor}

\subsubsection{Redes científicas}

Participação do autor nas redes científicas e seus respectivos endereços eletrônicos, onde o autor registra e disponibiliza suas publicações.

\begin{tabular}{ll}
\multicolumn{1}{c}{ Rede cientifica } & \multicolumn{1}{c}{ Link para acesso ao perfil } \\
ORCID & https://orcid.org/0000-0003-2783-3072 \\
Lattes & http://lattes.cnpq.br/1998963751735894 \\
https://www.scopus.com/authid/detail.uri?origin=resultslist\&authorld= \\
ScopusID & $11839167000 \& z o n e=$ \\
Publons/ResearcherID & - \\
ResearchGate & https://www.researchgate.net/profile/Raffael_Zamper3 \\
Google Schoolar & https://scholar.google.com.br/citations?user=MwhorH8AAAAJ\&hl=pt- \\
& PT\&oi=sra \\
Academia.edu & https://independent.academia.edu/RAFFAELZAMPER \\
\hline
\end{tabular}




\subsubsection{Trabalhos publicados}

Trabalhos publicados e indexados na base de dados referencial Web of

Science.

\begin{tabular}{|c|c|c|c|c|}
\hline \multicolumn{5}{|c|}{ Publicações } \\
\hline Ano & FI & Categoria & Referência & Citações \\
\hline 2010 & 0.968 & Article & $\begin{array}{l}\text { Zamper RP, Torres ML, Ferraz JL, Mori } \\
\text { Neto S, Holzhacker R, Shimada V, et al. } \\
\text { Evaluation of a computerized anesthesia } \\
\text { report. Rev Bras Anestesiol. } \\
2010 ; 60(3): 285-301 .\end{array}$ & 1 \\
\hline 2017 & Sem FI & Article & $\begin{array}{l}\text { Zamper RP, Amorim TC, Costa LG, } \\
\text { Takaoka F, Serpa Neto A. The role of } \\
\text { thromboelastometry in the assessment } \\
\text { and treatment of coagulopathy in liver } \\
\text { transplant patients. einstein (Sao Paulo). } \\
2017 ; 15(2): 243-6 \text {. }\end{array}$ & 1 \\
\hline 2018 & 1.619 & Article & $\begin{array}{l}\text { Zamper RP, Amorim TC, Queiroz VN, } \\
\text { Lira JD, Costa LG, Takaoka F, } \\
\text { Juffermans NP, Serpa Neto A. } \\
\text { Association between viscoelastic tests- } \\
\text { guided therapy with synthetic factor } \\
\text { concentrates and allogenic blood } \\
\text { transfusion in liver transplantation: a } \\
\text { before-after study. BMC Anesthesiol. } \\
\text { 2018;18(1):198. }\end{array}$ & 1 \\
\hline 2019 & 3.000 & Article & $\begin{array}{l}\text { Yokoyama AP, Kutner JM, Sakashita } \\
\text { AM, Nakazawa CY, de Paula TA, } \\
\text { Zamper RP, et al. Risk factors for } \\
\text { transfusion after orthotopic liver } \\
\text { transplantation. Transfus Med Hemother. } \\
\text { 2019;46(6):431-9. }\end{array}$ & 0 \\
\hline 2020 & 0.968 & Case report & $\begin{array}{l}\text { Zamper RP, Bainbridge D, Nagpal D, } \\
\text { Fujii S. Conduta em obstrução por } \\
\text { coágulo em cânula de duplo lúmen } \\
\text { bicaval após diagnóstico guiado por } \\
\text { ETE: relato de caso. Rev Bras } \\
\text { Anestesiol. In press } 2020 \text {. }\end{array}$ & 0 \\
\hline
\end{tabular}

7.3.3 Mediana do fator de impacto dos trabalhos publicados

Fator de impacto das publicações

\begin{tabular}{|c|}
\hline 0 \\
\hline 0.968 \\
\hline 0.968 \\
\hline 1.619 \\
\hline 3.000 \\
\hline
\end{tabular}

\section{Mediana}

\subsection{8}




\subsubsection{Citações recebidas}

\begin{tabular}{|ccc|}
\hline \multicolumn{3}{|c|}{ Publicações $\mathbf{x}$ Citações } \\
\hline Ano & Publicações & Citações \\
\hline 2010 & 1 & 1 \\
\hline 2017 & 1 & 1 \\
\hline 2018 & 1 & 1 \\
\hline 2019 & 1 & 0 \\
\hline 2020 & 1 & 0 \\
\hline Total & $\mathbf{5}$ & $\mathbf{3}$ \\
\hline
\end{tabular}

\subsubsection{H índex e m quociente}

\begin{tabular}{|lc|}
\multicolumn{1}{c|}{ Cálculo do h-index } \\
\hline \multicolumn{1}{|c|}{ Tópicos } & Dados \\
\hline Quantidade de publicações: & 5 \\
\hline Soma do número de citações: & 3 \\
\hline Artigos que receberam citações: & 3 \\
\hline Média de citações por item: & 0,6 \\
\hline h-index : & $\mathbf{1}$ \\
\hline
\end{tabular}

\section{Cálculo do m-quociente}

h-index

1

Número de anos de publicação 


\begin{tabular}{|c|c|c|c|c|}
\hline \multicolumn{5}{|c|}{ Publicações - $\boldsymbol{h}$-index } \\
\hline Ano & FI & Categoria & Referência & Citações \\
\hline 2010 & 0.968 & Article & $\begin{array}{l}\text { Zamper RP, Torres ML, Ferraz JL, Mori Neto S, } \\
\text { Holzhacker R, Shimada V, et al. Evaluation of a } \\
\text { computerized anesthesia report. Rev Bras } \\
\text { Anestesiol. 2010;60(3):285-301. }\end{array}$ & 3 \\
\hline 2017 & $\begin{array}{c}\text { Sem } \\
\text { FI }\end{array}$ & Article & $\begin{array}{l}\text { Zamper RP, Amorim TC, Costa LG, Takaoka F, } \\
\text { Serpa Neto A. The role of thromboelastometry } \\
\text { in the assessment and treatment of } \\
\text { coagulopathy in liver transplant patients. } \\
\text { einstein (Sao Paulo). } 2017 ; 15(2): 243-6 .\end{array}$ & 1 \\
\hline 2018 & 1.619 & Article & $\begin{array}{l}\text { Zamper RP, Amorim TC, Queiroz VN, Lira JD, } \\
\text { Costa LG, Takaoka F, Juffermans NP, Serpa } \\
\text { Neto A. Association between viscoelastic tests- } \\
\text { guided therapy with synthetic factor } \\
\text { concentrates and allogenic blood transfusion in } \\
\text { liver transplantation: a before-after study. BMC } \\
\text { Anesthesiol. 2018;18(1):198. }\end{array}$ & 1 \\
\hline 2019 & 3.000 & Article & $\begin{array}{l}\text { Yokoyama AP, Kutner JM, Sakashita AM, } \\
\text { Nakazawa CY, de Paula TA, Zamper RP, et al. } \\
\text { Risk factors for transfusion after orthotopic liver } \\
\text { transplantation. Transfus Med Hemother. } \\
\text { 2019;46(6):431-9. }\end{array}$ & 0 \\
\hline 2020 & 0.968 & Case report & $\begin{array}{l}\text { Zamper RP, Bainbridge D, Nagpal D, Fujii S. } \\
\text { Conduta em obstrução por coágulo em cânula } \\
\text { de duplo lúmen bicaval após diagnóstico guiado } \\
\text { por ETE: relato de caso. Rev Bras Anestesiol. In } \\
\text { press } 2020 \text {. }\end{array}$ & 0 \\
\hline
\end{tabular}




\section{REFERÊNCIAS}

1. Rana A, Petrowsky H, Hong JC, Agopian VG, Kaldas FM, Farmer D, et al. Blood transfusion requirement during liver transplantation is an important risk factor for mortality. J Am Coll Surg. 2013;216(5):902-7.

2. Maxwell MJ, Wilson MJ. Complications of blood transfusion. Contin Educ Anaesth Crit Care Pain. 2006;6:225-9.

3. Romero FA, Razonable RR. Infections in liver transplant recipients. World J Hepatol. 2011;3(4):83-92.

4. Massicotte L, Sassine MP, Lenis S, Roy A. Transfusion predictors in liver transplant. Anesth Analg. 2004;98(5):1245-51.

5. de Boer MT, Christensen MC, Asmussen M, van der Hilst CS, Hendriks HG, Slooff MJ, et al. The impact of intraoperative transfusion of platelets and red blood cells on survival after liver transplantation. Anesth Analg. 2008;106(1):32-44.

6. Liu S, Fan J, Wang X, Gong Z, Wang S, Huang L, et al. Intraoperative cryoprecipitate transfusion and its association with the incidence of biliary complications after liver transplantation—a retrospective cohort study. PLoS One. 2013;8(5):e60727.

7. Ramos E, Dalmau A, Sabate A, Lama C, Llado L, Figueras J, et al. Intraoperative red blood cell transfusion in liver transplantation: influence on patient outcome, prediction of requirements, and measures to reduce them. Liver Transpl. 2003;9(12):1320-7.

8. Cacciarelli TV, Keeffe EB, Moore DH, Burns W, Busque S, Concepcion W, et al. Effect of intraoperative blood transfusion on patient outcome in hepatic transplantation. Arch Surg. 1999;134(1):25-9.

9. Goodnough LT, Brecher ME, Kanter $\mathrm{H}$, et al. Transfusion medicine - blood transfusion - first of two parts. N Engl J Med. 1999;340:438-47.

10. Goodnough LT, Brecher ME, Kanter H, et al. Transfusion medicine - blood conservation second of two parts. N Engl J Med. 1999;340:525-33.

11. Spahn DR, Casutt M. Eliminating blood transfusions: new aspects and perspectives. Anesthesiology. 2000;93(1):242-55.

12. Saner FH, Gieseler RK, Akız H, Canbay A, Gorlinger K. Delicate balance of bleeding and thrombosis in end-stage liver disease and liver transplantation. Digestion. 2013;88(3):135-44.

13. Agarwal A, Sharma N, Vij V. Point-of-care coagulation monitoring during liver transplantation. Trends in Anaesthesia and Critical Care. 2013;3:42-8.

14. Blasi A, Beltran J, Pereira A, Martinez-Palli G, Torrents A, Balust J, et al. An assessment of thromboelastometry to monitor blood coagulation and guide transfusion support in liver transplantation. Transfusion. 2012;52(9):1989-98.

15. McCluskey SA, Karkouti K, Wijeysundera DN, Kakizawa K, Ghannam M, Hamdy A, et al. Derivation of a risk index for the prediction of massive blood transfusion in liver transplantation. Liver Transpl. 2006;12(11):1584-93. 
16. Ganter MT, Hofer CK. Coagulation monitoring: current techniques and clinical use of viscoelastic point-of-care coagulation devices. Anesth Analg. 2008;106(5):1366-75.

17. Luddington RJ. Thrombelastography/thromboelastometry. Clin Lab Haematol. 2005;27(2):81-90.

18. Kang YG, Martin DJ, Marquez J, Lewis JH, Bontempo FA, Shaw BW Jr, et al. Intraoperative changes in blood coagulation and thrombelastographic monitoring in liver transplantation. Anesth Analg. 1985;64(9):888-96.

19. Weber CF, Gorlinger K, Meininger D, Herrmann E, Bingold T, Moritz A, et al. Point-of-care testing: a prospective, randomized clinical trial of efficacy in coagulopathic cardiac surgery patients. Anesthesiology. 2012;117(3):531-47.

20. Davenport R, Khan S. Management of major trauma haemorrhage: treatment priorities and controversies. Br J Haematol. 2011;155(5):537-48.

21. Wang SC, Shieh JF, Chang KY, Chu YC, Liu CS, Loong CC, et al. Thromboelastographyguided transfusion decreases intraoperative blood transfusion during orthotopic liver transplantation: randomized clinical trial. Transplant Proc. 2010;42(7):2590-3.

22. Clevenger B, Mallett SV. Transfusion and coagulation management in liver transplantation. World J Gastroenterol. 2014;20(20):6146-58.

23. Tripodi A, Salerno F, Chantarangkul V, Clerici M, Cazzaniga M, Primignani M, et al. Evidence of normal thrombin generation in cirrhosis despite abnormal conventional coagulation tests. Hepatology. 2005;41(3):553-8.

24. Tripodi A, Primignani M, Chantarangkul V, Clerici M, Dell'Era A, Fabris F, et al. Thrombin generation in patients with cirrhosis: the role of platelets. Hepatology. 2006;44(2):440-5.

25. Tripodi A, Primignani M, Mannucci PM, Caldwell SH. Changing Concepts of Cirrhotic Coagulopathy. Am J Gastroenterol. 2017;112(2):274-81.

26. Hartmann M, Szalai C, Saner FH. Hemostasis in liver transplantation: Pathophysiology, monitoring, and treatment. World J Gastroenterol. 2016;22(4):1541-50.

27. Sahu S, Hemlata, Verma A. Adverse events related to blood transfusion. Indian J Anaesth. 2014;58(5):543-51.

28. Mannucci PM, Tripodi A. Liver disease, coagulopathies and transfusion therapy. Blood Transfus. 2013;11(1):32-6.

29. Yuasa T, Niwa N, Kimura S, Tsuji H, Yurugi K, Egawa H, et al. Intraoperative blood loss during living donor liver transplantation: an analysis of 635 recipients at a single center.

Transfusion. 2005;45(6):879-84.

30. Dunn LK, Thiele RH, Ma JZ, Sawyer RG, Nemergut EC. Duration of red blood cell storage and outcomes following orthotopic liver transplantation. Liver Transpl. 2012;18(4):475-81.

31. Hartert $\mathrm{H}$. Blutgerinnungstudien mit der Thrombelastographie, einem neuen Untersuchungsverfahren. Klin Wochenschr. 1948;26:557-83.

32. Hett DA, Walker D, Pilkington SN, Smith DC. Sonoclot analysis. Br J Anaesth. 1995;75(6):771-6. 
33. Muller MC, Meijers JC, Vroom MB, Juffermans NP. Utility of thromboelastography and/or thromboelastometry in adults with sepsis: a systematic review. Crit Care. 2014;18(1):R30.

34. Hanke AA, Herold U, Dirkmann D, Tsagakis K, Jakob H, Görlinger K. Thromboelastometry Based Early Goal-Directed Coagulation Management Reduces Blood Transfusion Requirements, Adverse Events, and Costs in Acute Type A Aortic Dissection: A Pilot Study. Transfus Med Hemother. 2012;39(2):121-8.

35. Lier H, Bottiger BW, Hinkelbein J, Krep H, Bernhard M. Coagulation management in multiple trauma: a systematic review. Intensive Care Med. 2011;37(4):572-82.

36. Gorlinger K, Fries D, Dirkmann D, Weber CF, Hanke AA, Schöchl H. Reduction of fresh frozen plasma requirements by perioperative point-of-care coagulation management with early calculated goal- directed therapy. Transfus Med Hemother. 2012;39(2):104-13.

37. Gorlinger K. [Coagulation management during liver transplantation]. Hamostaseologie. 2006;26(3 Suppl 1):S64-76. German.

38. Kirchner C, Dirkmann D, Treckmann JW, Paul A, Hartmann M, Saner FH, et al. Coagulation management with factor concentrates in liver transplantation: a single-center experience. Transfusion. 2014;54(10 Pt 2):2760-8.

39. Bell SF, Rayment R, Collins PW, Collis RE. The use of fibrinogen concentrate to correct hypofibrinogenaemia rapidly during obstetric haemorrhage. Int J Obstet Anesth. 2010;19(2):218-23.

40. Karlsson M, Ternstrom L, Hyllner M, Baghaei F, Flinck A, Skrtic S, et al. Prophylactic fibrinogen infusion reduces bleeding after coronary artery bypass surgery. A prospective randomised pilot study. Thromb Haemost. 2009;102(1):137-44.

41. Schochl H, Nienaber U, Hofer G, Voelckel W, Jambor C, Scharbert G, et al. Goal-directed coagulation management of major trauma patients using thromboelastometry (ROTEM)-guided administration of fibrinogen concentrate and prothrombin complex concentrate. Crit Care. 2010;14(2):R55.

42. Sorensen B, Bevan D. A critical evaluation of cryoprecipitate for replacement of fibrinogen. Br J Haematol. 2010;149(6):834-43.

43. Chowdary P, Saayman AG, Paulus U, Findlay GP, Collins PW. Efficacy of standard dose and $30 \mathrm{ml} / \mathrm{kg}$ fresh frozen plasma in correcting laboratory parameters of haemostasis in critically ill patients. Br J Haematol. 2004;125(1):69-73.

44. Rahe-Meyer N, Sørensen B. For: fibrinogen concentrate for management of bleeding. J Thromb Haemost. 2011;9(1):1-5.

45. Palomo JC, Jimenez C, Moreno E, Garcia MA, Bercedo J, Loinaz C, et al. Effects of intraoperative blood transfusion on rejection and survival after orthotopic liver transplantation. Transplant Proc. 1995;27(4):2326-7.

46. Janatpour K, Holland PV. Noninfectious serious hazards of transfusion. Curr Hematol Rep. 2002;1(2):149-55.

47. Nascimento B, Goodnough LT, Levy JH. Cryoprecipitate therapy. Br J Anaesth. 2014;113(6):922-34. 
48. Novakovic Anucin S, Kosanovic D, Gnip S, Canak V, Cabarkapa V, Mitic G. Comparison of standard coagulation tests and rotational thromboelastometryfor hemostatic system monitoring during orthotopic liver transplantation - results from a pilot study. Med Pregl. 2015;68(910):301-7.

49. Sabate A, Dalmau A. Fibrinogen: a clinical update on liver transplantation. Transplant Proc. 2015;47(10):2925-8.

50. Coakley M, Reddy K, Mackie I, Mallett S. Transfusion triggers in orthotopic liver transplantation: a comparison of the thromboelastometry analyzer, the thromboelastogram, and conventional coagulation tests. J Cardiothorac Vasc Anesth. 2006;20(4):548-53.

51. Pereboom IT, de Boer MT, Haagsma EB, Hendriks HG, Lisman T, Porte RJ. Platelet transfusion during liver transplantation is associated with increased postoperative mortality due to acute lung injury. Anesth Analg. 2009;108(4):1083-91.

52. Roullet S, Pillot J, Freyburger G, Biais M, Quinart A, Rault A, et al. Rotation thromboelastometry detects thrombocytopenia and hypofibrinogenaemia during orthotopic liver transplantation. Br J Anaesth. 2010;104(4):422-8.

53. Sarode R, Milling TJ Jr, Refaai MA, Mangione A, Schneider A, Durn BL, et al. Efficacy and safety of a 4-factor prothrombin complex concentrate in patients on vitamin $\mathrm{K}$ antagonists presenting with major bleeding: a randomized, plasma-controlled, phase Illb study. Circulation. 2013;128(11):1234-43.

54. Tanaka KA, Mazzeffi M, Durila M. Role of prothrombin complex concentrate in perioperative coagulation therapy. J Intensive Care. 2014;2(1):60.

55. Hanke AA, Joch C, Gorlinger K. Long-term safety and efficacy of a pasteurized nanofiltrated prothrombin complex concentrate (Beriplex $\mathrm{P} / \mathrm{N}$ ): a pharmacovigilance study. $\mathrm{Br} \mathrm{J}$ Anaesth. 2013;110(5):764-72.

56. Abuelkasem E, Hasan S, Mazzeffi MA, Planinsic RM, Sakai T, Tanaka KA. Reduced Requirement for prothrombin complex concentrate for the restoration of thrombin generation in plasma from liver transplant recipients. Anesth Analg. 2017;125(2):609-15.

57. Roullet S, Freyburger G, Cruc M, Quinart A, Stecken L, Audy M, et al. Management of bleeding and transfusion during liver transplantation before and after the introduction of a rotational thromboelastometry-based algorithm. Liver Transpl. 2015;21(2):169-79.

58. Song JG, Jeong SM, Jun IG, Lee HM, Hwang GS. Five-minute parameter of thromboelastometry is sufficient to detect thrombocytopenia and hypofibrinogenaemia in patients undergoing liver transplantation. Br J Anaesth. 2014;112(2):290-7.

59. Roullet S, Freyburger G, Labrouche S, Morisse E, Stecken L, Quinart A, et al. Hyperfibrinolysis during liver transplantation is associated with bleeding. Thromb Haemost. 2015;113(5):1145-8.

60. Xia VW, Steadman $\mathrm{RH}$. Antifibrinolytics in orthotopic liver transplantation: current status and controversies. Liver Transpl. 2005;11(1):10-8.

61. Makwana J, Paranjape S, Goswami J. Antifibrinolytics in liver surgery. Indian J Anaesth. 2010;54(6):489-95. 
62. Poon KS, Chen CC, Thorat A, Chiang YY, Jeng LB, Yang HR, et al. Fibrinolysis after reperfusion of liver graft. Acta Anaesthesiol Taiwan. 2015;53(1):41-3.

63. Bolliger D, Seeberger MD, Tanaka KA. Principles and practice of thromboelastography in clinical coagulation management and transfusion practice. Transfus Med Rev. 2012;26(1):1-13.

64. Blasi A. Coagulopathy in liver disease: lack of an assessment tool. World J Gastroenterol. 2015;21(35):10062-71. 


\section{Abstract}

Introduction: Perioperative bleeding and transfusion are important causes of morbidity and mortality in patients undergoing liver transplantation. Purpose: To assess whether viscoelastic tests-guided therapy with the use of synthetic factor concentrates impact transfusion rates of hemocomponents in adult patients undergoing liver transplantation. Methods: This is an interventional before-after comparative study. Patients undergoing liver transplantation before the implementation of a protocol using thromboelastometry and synthetic factor concentrates were compared to patients after the implementation. Primary outcome was transfusion of any hemocomponents. Secondary outcomes included: transfusion of red blood cells, fresh frozen plasma, cryoprecipitate or platelets, clinical complications, length of stay and in-hospital mortality. Results: A total of 183 patients were included in the control and 54 in the intervention phase. After propensity score matching, the proportion of patients receiving any transfusion of hemocomponents was lower in the intervention phase (37.0 versus $58.4 \%$; OR, 0.42 ; $95 \% \mathrm{Cl}, 0.20-0.87 ; \mathrm{p}=0.019)$. Patients in the intervention phase received less RBC (30.2 versus $52.5 \%$; OR, $0.21 ; 95 \% \mathrm{Cl}, 0.08-0.56 ; \mathrm{p}=0.002)$ and $\mathrm{FFP}(5.7$ versus $27.3 \%$; OR, $0.11 ; 95 \% \mathrm{Cl}, 0.03-0.43 ; \mathrm{p}=0.002$ ). There was no difference regarding transfusion of cryoprecipitate and platelets, complications related to the procedure, hospital length of stay and mortality. Conclusions: Use of a viscoelastic test-guided transfusion algorithm with the use of synthetic factor concentrates reduces the transfusion rates of allogenic blood in patients submitted to liver transplantation.

Keywords: Liver transplantation; Blood coagulation disorders; Blood transfusion; Hemostasis; Thrombelastography 